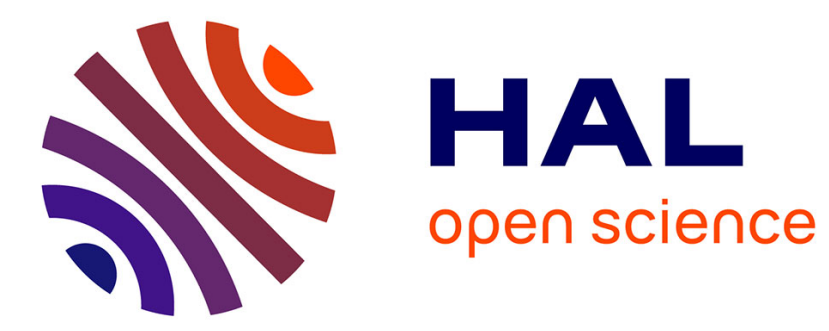

\title{
The $\ell$-distribution method for modeling non-gray absorption in uniform and non-uniform gaseous media
}

Frédéric André

\section{To cite this version:}

Frédéric André. The $\ell$-distribution method for modeling non-gray absorption in uniform and nonuniform gaseous media. Journal of Quantitative Spectroscopy and Radiative Transfer, 2016, 179, pp.19-32. 10.1016/j.jqsrt.2016.02.034 . hal-02064931

\section{HAL Id: hal-02064931 \\ https://hal.science/hal-02064931}

Submitted on 23 Apr 2019

HAL is a multi-disciplinary open access archive for the deposit and dissemination of scientific research documents, whether they are published or not. The documents may come from teaching and research institutions in France or abroad, or from public or private research centers.
L'archive ouverte pluridisciplinaire HAL, est destinée au dépôt et à la diffusion de documents scientifiques de niveau recherche, publiés ou non, émanant des établissements d'enseignement et de recherche français ou étrangers, des laboratoires publics ou privés. 


\title{
THE $\ell$-DISTRIBUTION METHOD FOR MODELING NON- GRAY ABSORPTION IN UNIFORM AND NON-UNIFORM GASEOUS MEDIA
}

\author{
Frédéric André
}

Université de Lyon, CNRS

INSA-Lyon, CETHIL, UMR5008, F-69621, Villeurbanne, France

Université Lyon 1, CETHIL, UMR5008, F-69622, France 
ABSTRACT. The -distribution modeling is proposed for radiative heat transfer in uniform and nonuniform non-gray gaseous media. The method is partly based on the application of results from the $k$ moment method. It combines this technique with several concepts from probability theory: the notion of rank transmutation maps allows extending the $k$-moment method to an infinite number of $k$ moments; copula models appear naturally to extend the method from uniform to non-uniform gas paths. The $\ell$-distribution approach is shown to provide results: $1 /$ more accurate - up to three orders of magnitude - than usual $k$-distribution approaches in uniform media, 2/ as precise as Correlated- $k$ models in non-uniform situations. All these results are obtained at a computational cost lower than $k$ distribution models. Differences and similarities between $k$ - and $\ell$-distribution methods are discussed.

KEYWORDS: e-distribution, rank transmutation mapping, Archimedean copulas, Path Dependent Scaling approximation. 


\section{NOMENCLATURE}

$c \quad$ copula density $c(X, Y)=\frac{\partial^{2} C(X, Y)}{\partial X \partial Y},(X, Y) \in[0,1]^{2}$

C copula

F distribution

$g \quad$ "germ" random variable; cumulative- $k$ distribution in Section 4.3.2.

$G r$ rank transmutation map in Section 3; defined as $G r=T_{R,\langle 2\rangle}$

$k \quad$ absorption coefficient $\left(\mathrm{cm}^{-1}\right)$

$l(\xi)$ random absorption length $(\mathrm{cm})$

$L \quad$ gas path length $(\mathrm{cm})$

$\mathbb{P} \quad$ probability law

$T_{R} \quad$ rank transmutation map in Section 2

\section{Greek symbols}

$\alpha \quad$ absorptivity as defined by Eq. (1)

$\beta \quad$ overlapping parameter as defined by Eqs. (14-b,39)

$\kappa_{\eta} \quad$ spectral absorption coefficient $\left(\mathrm{cm}^{-1}\right)$

$\lambda \quad$ inverse $k$-moment absorptivity model, Eqs. (12-13) (cm)

$\Lambda \quad$ in section $3, \Lambda=\lambda_{\langle 2\rangle}(\mathrm{cm})$

$\eta \quad$ wavenumber $\left(\mathrm{cm}^{-1}\right)$

$\tau \quad$ transmission function; transmissivity

$\Omega \quad$ natural logarithm of $1-\alpha^{\Delta \eta}(L)-$ see Eq. (7)

$\omega \quad$ coefficients in the series expansion of $\Omega$ and its reciprocal - Eq. (7)

$\xi \quad$ random variable uniformly distributed over $[0,1]$

\section{Subscripts}

$P \quad$ Planck mean

$R \quad$ Rosseland mean

$\langle n\rangle \quad$ related to the $k$-moment model at order $n$

$n \quad$ related to the $n$-th uniform sub-path along a non-uniform path 
1..n related to the $L_{1}+L_{2}+. .+L_{n}$ non-uniform path

\section{Superscripts}

$\Delta \eta \quad$ width of the spectral interval for the averaging of spectral properties 


\section{INTRODUCTION}

Many approximate models were developed during the past decades to treat radiative heat transfer in gaseous media [1,2]. The so-called $k$-moment approach is among the most recent ones. It was originally proposed in 2006 [3] and studied during the last ten years [4,5]. This paper is devoted - in part - to a reformulation and analysis of some useful results related to this technique. They are then used to propose a new approximate model for non-gray absorption in uniform and non-uniform gaseous media.

Probably the most difficult thing to understand in the $k$-moment approach is that its primary objective is not to derive $k$-distribution functions but absorptivities or transmissivities of gaseous paths. However, in some situations, the inverse Laplace transform of these transmissivities can be evaluated analytically and $k$-distributions can be obtained. Nevertheless, it is a consequence of the results of the method, not its main objective.

The $k$-moment approach is mostly funded on the idea that by using the moments of the absorption coefficient - that can be evaluated by many different ways - one can derive reliable and accurate approximate transmissivity or absorptivity models. With only two moments, for instance, it is possible to estimate all the quantities required for the application of the Statistical Narrow Band (SNB) model based on Malkmus' distribution of line-strengths [6]. However, many test cases showed that using only two moments with Malkmus-SNB is not sufficient to ensure accurate results for all possible thermophysical states of the gas and values of path-lengths - this may also explain why most Malkmus-SNB model parameters are obtained through adjustments on curves-of-growth [7], and not through their definition -. Several attempts - based for instance on the application of the Maximum Entropy principle, see [5] - were made during the past years to increase the number of moments used in the approximate models, viz. to add more than two moments to the Malkmus-SNB or to other possible models. However, no "perfect" solution was found up to now.

The aim of the present work is to show how results from the $k$-moment approach can be used as starting points: $1 /$ to propose models that can achieve LBL accuracy, $2 /$ to extend these models to nonuniform situations. It is based on the following ideas.

Mathematically, and under some restrictions, absorptivities are distribution functions. The corresponding random variable is an absorption length, hence the name of the new method for radiative heat transfer in non-uniform gaseous media proposed in this work: the l-distribution modeling.

The key idea of $\ell$-distribution modeling is the following one. As soon as absorptivities are regarded as distribution functions, the treatment of non-uniform paths can be reformulated equivalently into a new problem: how to derive a multi-variate distribution function - absorptivity or transmissivity of a nonuniform path - from its marginals - radiative properties over uniform paths -? This issue can be handled mathematically using Copula's theory. Using a probabilistic framework also allows using some mathematical tools, such as the concept of rank transmutation mapping. This provides a simple and accurate method to extend the $k$-moment approach to an infinite number of moments of the absorption coefficient.

The paper is structured as follows. The first section is dedicated to the development of the $\ell$-distribution modeling in uniform media: the principle of the model is described and the approach is connected with the $k$-moment method. Then, the principle of rank transmutation maps (RTMs) is introduced and an explicit formula for RTMs is derived by application of some results from the $k$-moment approach. The second section is devoted to the extension of the model to non-uniform media. For this purpose, the socalled path dependent scaling (PDS) approximation is proposed and detailed in the case of two uniform sub-paths. It is shown that this assumption can be formulated in terms of Archimedean copulas. The technique is then extended to any non-uniform path, using some mathematical properties of this particular type of copulas. This leads to the development of an iterative process, called the ICE (Iterative Copula Estimation) scheme, to treat any non-uniform path.

Although the method is sophisticated from a pure mathematical perspective, its use in radiative transfer is straightforward. Some test cases - including a Turbulence-Radiation Interaction problem known to be challenging from a computational perspective due to the large amount of calculations it requires - 
show that the present approach is at the same time as accurate and less computationally expensive that the more usual $k$-distribution technique.

\section{2. ८-DISTRIBUTIONS IN UNIFORM MEDIA}

\subsection{Principle of $\ell$-distributions}

The concept of distribution function is omnipresent in gas radiation modeling. It appears explicitly in the so-called $k$-distribution approach [2] but also in Statistical Narrow Band (SNB) models for which line positions and strengths are considered as random quantities with given distribution functions - spectral line centers are assumed to be uniformly distributed over narrow bands for instance $[1,2]$-.

The mathematical definition of a distribution - see Ref. [8], p.20 - is any non decreasing function $F$ on $\mathbb{R}$ having $F(-\infty)=0$ and $F(+\infty)=1$. For any such function, there exists - [8], p. 107 - a probability space and a random variable $X$ - defined in probability theory as a measurable function from this space to some real interval - for which the distribution function of $X$ is $F$ ie. $F(x)=\mathbb{P}(X \leq x)$ where $-\infty<x<+\infty$.

Following this definition - and restricting it to positive real numbers -, the absorptivity of a uniform path of length $L$ inside a gas averaged over a narrow spectral band defined as:

$$
\alpha^{\Delta \eta}(L)=1-\frac{1}{\Delta \eta} \int_{\Delta \eta} \exp \left(-\kappa_{\eta} L\right) d \eta=\mathbb{P}[l(\xi) \leq L]
$$

is a $\ell$-distribution as soon as the spectral interval considered for the averaging does not contain any transparency region of the gas - this constraint is required in order to ensure that the absorptivity takes value 1 for infinitely long paths. This result applies to any band width up to the full spectrum but a weighting by the Planck function may be required. It can be noticed that transmissivities can also be interpreted as survival distributions ie. $\tau^{\Delta \eta}(L)=1-\alpha^{\Delta \eta}(L)=\mathbb{P}[l(\xi)>L]$.

The corresponding random variable $l(\xi)$ - that represents an absorption path length - in Eq. (1) can be defined as the solution of the implicit equation:

$$
\xi=\alpha^{\Delta \eta}[l(\xi)]=1-\frac{1}{\Delta \eta} \int_{\Delta \eta} \exp \left[-\kappa_{\eta} l(\xi)\right] d \eta
$$

where $\xi$ is a random variable uniformly distributed over [0,1]. This is a direct consequence of the so-called inverse transformation theorem ([8], p. 111), widely used in Monte Carlo approaches [9]. Let us now consider any arbitrary approximate model $\alpha_{*}^{\Delta \eta}(L)$ for $\alpha^{\Delta \eta}(L)$. The minimum constraint that we can impose to $\alpha_{*}^{\Delta \eta}(L)$ is to coincide with $\alpha^{\Delta \eta}(L)$ for the two extreme values $L=0$ and $L=+\infty$. If in addition we impose $\alpha_{*}^{\Delta \eta}(L)$ to be a strictly monotonous function of $L$, then we can immediately observe that $\alpha_{*}^{\Delta \eta}(L)$ is also a distribution function. As for $\alpha^{\Delta \eta}(L)$, the corresponding random variable can be defined as solution of the following implicit equation:

$$
\xi=\alpha_{*}^{\Delta \eta}\left[l_{*}(\xi)\right]
$$

where again $\xi$ is uniformly distributed over $[0,1]$.

From the two previous definitions, it is obvious that random variables $l(\xi)$ and $l_{*}(\xi)$ take values inside the same interval, which is in this case $[0,+\infty]$ - notice that the previous interval is closed which means that infinite values of $l(\xi)$ and $l_{*}(\xi)$ are allowed -. 
For two distribution functions $F_{1}$ and $F_{2}$ with corresponding random variables that take values inside the same interval, Shaw and Buckley [10] define a rank transmutation mapping (RTM) function $T_{R}$ as:

$$
F_{1}^{-1}(\xi)=F_{2}^{-1}\left[T_{R}(\xi)\right] \Leftrightarrow T_{R}(\xi)=F_{2}\left[F_{1}^{-1}(\xi)\right]
$$

$T_{R}: 1 /$ is defined as the functional composition of the cumulative distribution function of one distribution with the inverse of the other one, 2/ maps the unit interval [0,1] into itself and connects the quantile - ie inverse - functions associated with the two distributions. Rank transmutation maps were introduced in [10] in order to modulate a given base distribution for the purpose of modifying its moments. It is a method to add moments to any base distribution in the same way as Edgeworth and Gram-Charlier series expansions, but without some deficiencies and problems in terms of convergence that may be encountered with these techniques - see Ref. [10] for more details -.

The same concept can be used in gas radiation modeling. Indeed, by replacing respectively $F_{1}$ and $F_{2}$ by $\alpha_{*}^{\Delta \eta}$ and $\alpha^{\Delta \eta}$ in the last equation, we can construct in our case the following RTM:

$$
T_{R}(\xi)=\alpha^{\Delta \eta}\left[\left(\alpha_{*}^{\Delta \eta}\right)^{-1}(\xi)\right]=\alpha^{\Delta \eta}\left[l_{*}(\xi)\right]
$$

Now, if we replace the random variable $\xi$ by the deterministic quantity $\alpha_{*}^{\Delta \eta}(L)$ in the previous relationship, we obtain:

$$
T_{R}\left[\alpha_{*}^{\Delta \eta}(L)\right]=\alpha^{\Delta \eta}(L)
$$

that is to say a possible way to write the true $\ell$-distribution $\alpha^{\Delta \eta}(L)$ in terms of its approximation $\alpha_{*}^{\Delta \eta}(L)$. The main problem consists in evaluating function $T_{R}$ but also to choose a base distribution. This problem is discussed in the next section where, by using some results from the $k$ moment method, we provide an explicit equation for the RTM function.

\subsection{The $k$-moment method}

The principle of the $k$-moment method was first described in Ref. [4], where it was formulated in terms of transmissivities and for any band width. Here, we will write it equivalently in terms of absorptivities, so as to comply with the definition of the RTM as given by Eq. (5). Moreover, in order to simplify the derivation, only narrow bands will be considered. The $k$-moment method can be explained as follows.

Let us consider a narrow spectral interval $\Delta \eta$ that: $H I /$ is narrow enough to assume that the Planck function remains constant, $H 2 /$ does not contain any transparency region of the gas at any temperature. For simplicity, we will first consider a single absorbing gas in a thermophysical state described by a state vector $\underline{\phi}$ that encompasses all the quantities required to characterize the path: its temperature, total pressure and molar fraction in absorbing species. The spectral absorption coefficient of the gas in state $\underline{\phi}$ is $\kappa_{\eta}$.

Let us define $\Omega(L)$ as the logarithm of $1-\alpha^{\Delta \eta}(L)$, where $\alpha^{\Delta \eta}(L)$ is given by Eq. (1). $\Omega(L)$ can be expanded as a Taylor-Lagrange series in the variable $L$ around the origin viz. $L=0$ to provide:

$$
\Omega(L)=\ln \left[1-\alpha^{\Delta \eta}(L)\right]=\omega_{0}+\omega_{1} L+\omega_{2} L^{2}+\ldots
$$


The first three coefficients $\omega$ are, by simply evaluating the derivatives of $\ln \left[1-\alpha^{\Delta \eta}(L)\right]$ with respect to $L$ at the origin $L=0$ :

$$
\begin{gathered}
\omega_{0}=\ln \left[1-\alpha^{\Delta \eta}(L=0)\right]=0 \\
\omega_{1}=\frac{\partial \ln \left[1-\alpha^{\Delta \eta}(L=0)\right]}{\partial L}=-\frac{1}{1-\alpha^{\Delta \eta}(L=0)} \frac{\partial \alpha^{\Delta \eta}(L=0)}{\partial L}=-\frac{1}{\Delta \eta} \int_{\Delta \eta} \kappa_{\eta} d \eta=-k_{P} \\
\omega_{2}=\frac{1}{2} \frac{\partial^{2} \ln \left[1-\alpha^{\Delta \eta}(L=0)\right]}{\partial L^{2}}=\frac{1}{2} \frac{1}{\Delta \eta} \int_{\Delta \eta}\left(\kappa_{\eta}-k_{P}\right)^{2} d \eta
\end{gathered}
$$

In the previous equations, $k_{P}$ is used to represent the (Planck) mean absorption coefficient of the gas over $\Delta \eta$.

Now, as $\omega_{0}=0$ and $\omega_{1}=-k_{P}<0$ following assumption $H 2$, we can conclude that the series expansion can be reversed to yield:

$$
L=\omega_{1}^{\prime} \Omega(L)+\omega_{2}^{\prime} \Omega(L)^{2}+\ldots
$$

in which the coefficients are given as - see for instance Ref. [11] for more details about series reversion formulas - :

$$
\begin{gathered}
\omega_{1}^{\prime}=\frac{1}{\omega_{1}}=-\frac{1}{k_{P}} \\
\omega_{2}^{\prime}=-\frac{\omega_{2}}{\omega_{1}^{3}}=\frac{1}{k_{P}} \frac{\omega_{2}}{\omega_{1}^{2}}
\end{gathered}
$$

According to the previous results, one can rewrite Eq. (9) under the following mathematical form:

$$
\begin{aligned}
& -k_{P} L=\Omega(L)-\frac{\omega_{2}}{\omega_{1}^{2}} \Omega(L)^{2}+\ldots=\ln \left[1-\alpha^{\Delta \eta}(L)\right]-\frac{\omega_{2}}{\omega_{1}^{2}}\left\{\ln \left[1-\alpha^{\Delta \eta}(L)\right]\right\}^{2}+\ldots \\
& \Leftrightarrow L=-\frac{\ln \left[1-\alpha^{\Delta \eta}(L)\right]}{k_{P}}+\frac{\omega_{2} k_{P}}{\omega_{1}^{2}}\left\{\frac{\ln \left[1-\alpha^{\Delta \eta}(L)\right]}{k_{P}}\right\}^{2}+\ldots
\end{aligned}
$$

Eq. (11) allows defining two sequences of functions $\lambda_{\langle n\rangle}, n \geq 1$ and $\alpha_{\langle n\rangle}^{\Delta \eta}(L), n \geq 1$ :

- functions $\lambda_{\langle n\rangle}, n \geq 1$ are lengths defined by truncating the series expansion at the RHS in Eq. (11) at some finite order $n$. This provides for instance for the first two orders:

$$
\begin{gathered}
\lambda_{\langle 1\rangle}(X)=-\frac{\ln (1-X)}{k_{P}} \\
\lambda_{\langle 2\rangle}(X)=-\frac{\ln (1-X)}{k_{P}}+\frac{\omega_{2} k_{P}}{\omega_{1}^{2}}\left[\frac{\ln (1-X)}{k_{P}}\right]^{2}
\end{gathered}
$$


- functions $\alpha_{\langle n\rangle}^{\Delta \eta}(L), n \geq 1$ are defined as the solutions of the set of functional equations, derived directly from Eq. (11):

$$
L=\lambda_{\langle n\rangle}\left[\alpha_{\langle n\rangle}^{\Delta \eta}(L)\right]
$$

Eq. (13) simply means that functions $\lambda_{\langle n\rangle}, n \geq 1$ are the inverse of $\alpha_{\langle n\rangle}^{\Delta \eta}(L), n \geq 1$.

Each solution $\alpha_{\langle n\rangle}^{\Delta \eta}(L), n \geq 1$ obtained by this technique corresponds to an approximate absorptivity model. The method to obtain such models consists in the following two step process - see Ref. [4] for more details and application of the technique up to order $n=3-: 1 /$ solve the polynomial equation for $\Omega(L)$ set by Eq. (9) for a given prescribed integer $n, 2 /$ retain, for orders $n$ higher than 1 , the solution that provides values of $\alpha_{\langle n\rangle}^{\Delta \eta}(L), L \geq 0$ inside the $[0,1]$ interval. This approach gives, for instance for the first two orders:

$$
\begin{gathered}
\alpha_{\langle 1\rangle}^{\Delta \eta}(L)=1-\exp \left(-k_{P} L\right) \\
\alpha_{\langle 2\rangle}^{\Delta \eta}(L)=1-\exp \left[-\frac{\beta}{\pi}\left(\sqrt{1+\frac{2 \pi k_{P} L}{\beta}}-1\right)\right], \frac{\beta}{\pi}=\frac{\omega_{1}^{2}}{2 \omega_{2}}
\end{gathered}
$$

Accordingly, the absorptivity model associated with the first order - Eq. (14-a) - corresponds to the gray gas approximation; the second order - Eq. (14-b) - provides a formulation that is similar to the widely used Statistical Narrow Band (SNB) model with Malkmus' distribution function of linestrengths [6].

Now, if we have a closer look at solutions (14-a) and (14-b) we can see that by increasing the order $n$ of the series expansion by 1 - starting from Eq. (14-a), viz. $n=1$ - allows adding in the corresponding absorptivity model - (14-b) - some new quantities that correspond to the coefficient added in the series expansion, that it to say information about the $n$-th moment - in the present case, information about the second moment, see Eq. (8-c) -. By increasing $n$ indefinitely, one can expect to converge toward the exact absorptivity given by Eq. (1) that obviously contains information about all the $k$-moments. This idea is basically the principle of the so-called $k$-moment method.

Most previous works on this approach stopped at this stage, viz. at order 2. Indeed, solving Eq. (13) becomes quickly complicated as $n$ increases. Consequently, formulation (14-b) was up to now the most studied in our previous works. It was used as the building block: 1/ to derive Curtis-Godson like approximations in non-uniform media in Refs. [12,13]; to propose an approximate model called the Spectral Line Moment Based model - over the full spectrum in Ref. [14]; etc. In the next section, we will show how the previous results can be used to provide explicit rank transmutation maps.

\subsection{Some relationships between the $k$-moments and $\ell$-distributions}

First of all, we can notice that if we combine Eq. (13) and Eq. (12-a) then, for any integer $n \geq 1$, Eq. (13) can be written in equivalent form:

$$
\underbrace{\ln \left[1-\alpha_{\langle 1\rangle}^{\Delta \eta}(L)\right]}_{=-k_{p} L}=-k_{p} \lambda_{\langle 1\rangle}\left[\alpha_{\langle 1\rangle}^{\Delta \eta}(L)\right]=-k_{p} \lambda_{\langle n\rangle}\left[\alpha_{\langle n\rangle}^{\Delta \eta}(L)\right]
$$


Then, Eq. (1), together with $\exp \left(-\kappa_{\eta} L\right)=\left[\exp \left(-k_{P} L\right)\right]^{\frac{\kappa_{\eta}}{k_{P}}}=\left[1-\alpha_{\langle 1\rangle}^{\Delta \eta}(L)\right]^{\frac{\kappa_{\eta}}{k_{P}}}-$ see Eq. (14-a) - , can be used to write:

$$
\alpha^{\Delta \eta}(L)=\frac{1}{\Delta \eta} \int_{\Delta \eta}\left[1-\exp \left(-\kappa_{\eta} L\right)\right] d \eta=1-\frac{1}{\Delta \eta} \int_{\Delta \eta}\left[1-\alpha_{\langle 1\rangle}^{\Delta \eta}(L)\right]^{\frac{\kappa_{\eta}}{k_{P}}} d \eta
$$

or equivalently, from Eq. (15) written as $1-\alpha_{\langle 1\rangle}^{\Delta \eta}(L)=\exp \left\{-k_{p} \lambda_{\langle n\rangle}\left[\alpha_{\langle n\rangle}^{\Delta \eta}(L)\right]\right\}$ :

$$
\left\{\begin{array}{l}
\alpha^{\Delta \eta}(L)=T_{R,\langle n\rangle}\left[\alpha_{\langle n\rangle}^{\Delta \eta}(L)\right] \\
T_{R,\langle n\rangle}(X)=1-\frac{1}{\Delta \eta} \int_{\Delta \eta} \exp \left[-\kappa_{\eta} \lambda_{\langle n\rangle}(X)\right] d \eta
\end{array}\right.
$$

This shows how to represent, for any integer $n \geq 1$, the true absorptivity $\alpha^{\Delta \eta}(L)$ as a function of its approximation $\alpha_{\langle n\rangle}^{\Delta \eta}(L)$. By comparing Eq. (17) and Eq. (6), we can conclude that as soon as the base distribution functions $\alpha_{*}^{\Delta \eta}$ is chosen as a member of the set of models obtained from the $k$ moment method, then the corresponding rank transmutation map takes the form given by Eq. (17).

Eqs. $(5,17)$ allow proposing a simple method to evaluate function $T_{R,\langle n\rangle}(X)$ for a given prescribed value of integer $n$ :

$1 /$ choose any random value $\xi$ uniformly distributed over $[0,1]$,

2/ apply function $\lambda_{\langle n\rangle}$, as defined for instance for the first two values of $n$ by Eqs. (12), to $\xi$ in order to obtain a length $l_{*}(\xi)=\lambda_{\langle n\rangle}(\xi)$,

3/ the corresponding value of the rank transmutation function is then simply, from Eq. (17):

$$
T_{R,\langle n\rangle}(\xi)=\alpha^{\Delta \eta}\left[\lambda_{\langle n\rangle}(\xi)\right]
$$

This method allows evaluating $T_{R,\langle n\rangle}$ directly from LBL data. The process can be iterated for a large number of samples $\xi$ and the corresponding values of $T_{R,\langle n\rangle}(\xi)$ stored in Tables. Then, $T_{R,\langle n\rangle}(X)$ can be estimated for any $X \in[0,1]$ by interpolating the data stored in these Tables. By choosing arbitrarily an integer $n$, one can thus propose the following simple scheme to approximate the absorptivity of a uniform gaseous medium:

A/ Model parameter generation:

$1 /$ use the $k$-moment solution at order $n$ to generate a base distribution function,

$2 /$ then, evaluate the corresponding rank transmutation map by using the method described previously. These values can be estimated for a given set of values of parameter $\xi$ - see Eq. (18) and then stored in Tables. More details about the method retained in this work will be provided later in this paper. 


\section{$\mathrm{B} /$ Application for the evaluation of gas radiative properties:}

3/ for a given length $L$ inside the gas, first evaluate a "base" absorptivity using the approximate $k$ moment model at order $n$ - see Step 1 -,

4/ then, map this value to the "real" one using the values of the rank transmutation mapping function stored in the Table.

It can be noticed that as soon as the value of parameter $X$ is one of the values of $\xi$ chosen to generate the Table, then applying the previous scheme provides the exact LBL solution. Indeed, using mapping functions mostly provides a convenient method to parameterize and interpolate LBL absorptivity data - this is the main signification of Eq. (17) -. But instead of tabulating these data over the $[0,+\infty]$ interval of possible values of the gas path lengths $L$, which would not be handy, the method proposed here allows reformulating the problem over the $[0,1]$ interval, using an approximate model for this purpose. This approach is thus very similar to the usual $k$-distribution method for which a high resolution spectrum is condensed into a small set of $k$-values and the wavenumber axis is, using a probabilistic framework, reduced to the $[0,1]$ interval.

We will from now on restrict our developments to the case $n=2$. The corresponding mapping and $\lambda$ functions will be written $\operatorname{Gr}(X)=T_{R,\langle 2\rangle}(X)$ - so as to comply with the notation proposed in Ref. [10], and since no confusion with a Grashof number can be made here - and $\Lambda(X)=\lambda_{\langle 2\rangle}(X)$ to avoid possible misunderstanding. Examples of functions $\operatorname{Gr}(X)=T_{R,\langle 2\rangle}(X)$ are shown in Figure 1.

\section{3.}

Some of the developments described in the previous section also have applications for the extension of the method to non-uniform gaseous media. This problem is the aim of the present section.

\subsection{Preliminary comments}

We consider now a non-uniform path discretized into $M$ uniform sub-paths. Each sub-path is characterized by a state vector $\underline{\phi}_{m}, m=1, \ldots, M$ and a length $L_{m}$. The transmissivity of a uniform path of length $L$ inside the gas in thermophysical state $\phi_{m}$ will be from now on written under the following form $\tau_{m}^{\Delta \eta}(L)$. Over each uniform path, we also define local functions $G r$ and $\Lambda$. As for transmissivities, an explicit dependency of these functions with respect to the local thermophysical state is required in order to avoid possible confusion. This dependency with respect to $\underline{\phi_{m}}, m=1, \ldots, M$ will be represented by an index $m$ - without \langle\rangle , which is used to represent a $k$ moment model at a given integer order - ie $G r_{m}$ and $\Lambda_{m}$.

Our objective is to extend the previous results, described for uniform situations, to this non-uniform path. We will start by studying in details the case $M=2$.

Let us thus consider a non-uniform path $L_{1}+L_{2}$ defined as the juxtaposition of a path of length $L_{1}$ inside the gas at $\underline{\phi}_{1}$ and a length $L_{2}$ inside the gas at $\underline{\phi}_{2}$. The band averaged transmissivity of the non-uniform path is in this case:

$$
\tau_{12}^{\Delta \eta}\left(L_{1}, L_{2}\right)=\frac{1}{\Delta \eta} \int_{\Delta \eta} \exp \left[-\kappa_{\eta}\left(\underline{\phi_{1}}\right) L_{1}-\kappa_{\eta}\left(\underline{\phi_{2}}\right) L_{2}\right] d \eta
$$


$\tau_{12}^{\Delta \eta}\left(L_{1}, L_{2}\right)$ can be interpreted as a joint probability $\tau_{12}^{\Delta \eta}\left(L_{1}, L_{2}\right)=\mathbb{P}\left[l_{1}\left(\xi_{1}\right)>L_{1}, l_{2}\left(\xi_{2}\right)>L_{2}\right]$ where random variables $l_{m}\left(\xi_{m}\right), m=1,2$ are defined as solutions of:

$$
\xi_{m}=\alpha_{m}^{\Delta \eta}\left[l_{m}\left(\xi_{m}\right)\right]=1-\frac{1}{\Delta \eta} \int_{\Delta \eta} \exp \left[-\kappa_{\eta}\left(\underline{\phi}_{m}\right) l_{m}\left(\xi_{m}\right)\right] d \eta, m=1,2
$$

In Eq. (20), $\xi_{m}, m=1,2$ are two independent random variables uniformly distributed over the $[0,1]$ interval.

The two random variables $l_{m}\left(\xi_{m}\right), m=1,2$ are not independent since:

$$
\begin{aligned}
\tau_{12}^{\Delta \eta}\left(L_{1}, L_{2}\right) & =\mathbb{P}\left[l_{1}\left(\xi_{1}\right)>L_{1}, l_{2}\left(\xi_{2}\right)>L_{2}\right] \\
& \neq=\underbrace{\frac{1}{\Delta \eta} \int_{\Delta \eta} \exp \left[-\kappa_{\eta}\left(\underline{\phi}_{1}\right) L_{1}\right] d \eta \frac{1}{\Delta \eta} \int_{\Delta \eta} \exp \left[-\kappa_{\eta}\left(\underline{\phi}_{2}\right) L_{2}\right] d \eta}_{=\mathbb{P}\left[l_{1}\left(\xi_{1}\right)>L_{1}\right] \mathbb{P}\left[l_{2}\left(\xi_{2}\right)>L_{2}\right]}
\end{aligned}
$$

However, it was shown by Sklar in Ref. [15] that one can find a bi-variate function $C$ - called a copula [16] - such that:

$$
\begin{aligned}
\mathbb{P}\left[l_{1}\left(\xi_{1}\right)>L_{1}, l_{2}\left(\xi_{2}\right)>L_{2}\right] & =C\left\{\mathbb{P}\left[l_{1}\left(\xi_{1}\right)>L_{1}\right], \mathbb{P}\left[l_{2}\left(\xi_{2}\right)>L_{2}\right]\right\} \\
& =C\left[\tau_{1}^{\Delta \eta}\left(L_{1}\right), \tau_{2}^{\Delta \eta}\left(L_{2}\right)\right]
\end{aligned}
$$

As $\tau_{1}^{\Delta \eta}\left(L_{1}\right)=\mathbb{P}\left[l_{1}\left(\xi_{1}\right)>L_{1}\right]$ and $\tau_{2}^{\Delta \eta}\left(L_{2}\right)=\mathbb{P}\left[l_{2}\left(\xi_{2}\right)>L_{2}\right]$ are continuous, the copula is unique. More details about copulas are given in Appendix A.

The main difficulty, when one wants to represent some dependency between random variables via a copula models, is to find which copula representation (Archimedean, Gaussian, etc) to use for a given application. In our case, this problem can be solved easily if gas spectra are assumed path dependent scaled. This concept, together with its mathematical formulation, is described in the next paragraphs.

\subsection{The Path Dependent Scaling (PDS) approximation}

\subsubsection{Mathematical formulation}

Two gas spectra will be called path dependent scaled if there exists a wavenumber independent real and positive coefficient $u$ - that depends on the thermophysical states of the gas, $\underline{\phi}_{1}$ and $\underline{\phi}_{2}$ associated with the two spectra and on the length of the path $L$. Notice that we will omit here the explicit dependency of $u$ with respect to the thermophysical states so as to abridge the notations such that for any length $L_{1}$ inside the gas in state $\phi_{1}$ :

$$
\frac{1}{\Delta \eta} \int_{\Delta \eta} \exp \left[-\kappa_{\eta}\left(\underline{\phi}_{1}\right) L_{1}\right] d \eta=\frac{1}{\Delta \eta} \int_{\Delta \eta} \exp \left[-\kappa_{\eta}\left(\underline{\phi}_{2}\right) u\left(L_{1}\right) L_{1}\right] d \eta
$$

In our previous works [17,18], this assumption was - improperly - referred to as the Scaled- $k$ approximation with reference to the implicit equation provided in Ref. [19]. However, in this reference, the objective is to obtain an effective scaling coefficient that is then fixed inside the 
medium. In our case, this quantity is allowed to depend on $L$ - see for instance Appendix B in Ref. [17] for a short discussion on this specific point -. Accordingly, physical assumptions are fundamentally different although they involve exactly the same implicit equation.

Indeed, physically, the PDS approximation extends the scaling approximation - for which $u$ does not depend on $L$ - by assuming that one can write the absorption coefficient in state $\underline{\phi}_{1}$ as:

$$
\kappa_{\eta}\left(\underline{\phi}_{1}\right)=\kappa_{\eta}\left(\underline{\phi_{2}}\right)\left(\bar{u}+\delta u_{\eta}\right)
$$

where $\bar{u}$ represents the value of the scaling function at the optically thin limit - it is the ratio of the Planck mean absorption coefficients - and $\delta u_{\eta}$ is a wavenumber dependent effect that measures the error made by approximating the spectra as actually scaled. In this case, it was shown in Ref. [17] - further details are also provided in Appendix B - that the effect of some errors in the scaling coefficient - viz. $\delta u_{\eta} \neq 0$ - is to induce a dependency of the effective scaling coefficient - i.e. the scaling coefficient that would provide the exact properties of the gas in state $\underline{\phi_{1}}$ - with respect to the length of the gas path. This dependency involves a length dependent scaling coefficient that can be obtained by solving the following implicit equation - equivalent to Eq. (23) - :

$$
\tau_{1}^{\Delta \eta}\left(L_{1}\right)=\frac{1}{\Delta \eta} \int_{\Delta \eta} \exp \left[-\kappa_{\eta}\left(\underline{\phi}_{2}\right) u\left(L_{1}\right) L_{1}\right] d \eta
$$

The PDS assumption share many similarities with the usual scaling. Indeed, in both cases, all the values of the absorption coefficient are assumed to follow rigorously the same trend - viz. nonuniformities affect all the values taken by the absorption coefficient in exactly the same way. In other words, this means that errors $\delta u_{\eta}$ are statistically independent of $\kappa_{\eta}\left(\phi_{2}\right)$ - which is in fact not true but is a simplifying as sumption. The accuracy of the PDS approximation will be assessed later, in the section dedicated to the application of the model.

Now, if gas spectra are assumed path dependent scaled, then one can approximate Eq. (19) as:

$$
\tau_{12}^{\Delta \eta}\left(L_{1}, L_{2}\right) \approx \frac{1}{\Delta \eta} \int_{\Delta \eta} \exp \left[-\kappa_{\eta}\left(\underline{\phi}_{2}\right)\left(L_{2}+u\left(L_{1}\right) L_{1}\right)\right] d \eta=\tau_{2}^{\Delta \eta}\left[L_{2}+u\left(L_{1}\right) L_{1}\right]
$$

Eq. (26), together with the following two relationships:

$$
\begin{gathered}
\tau_{2}^{\Delta \eta}\left(L_{2}\right)=\frac{1}{\Delta \eta} \int_{\Delta \eta} \exp \left[-\kappa_{\eta}\left(\underline{\phi}_{2}\right) L_{2}\right] d \eta \\
\tau_{1}^{\Delta \eta}\left(L_{1}\right)=\tau_{2}^{\Delta \eta}\left[u\left(L_{1}\right) L_{1}\right]
\end{gathered}
$$

defines a so-called Archimedean copula. More details about this particular type of copula, which are among the most widely encountered [16], are provided in Appendix A.

Basically, applying the non- uniform model set by Eqs. (26-28) consists in the following two steps illustrated in Figure 2-a -:

1/ first "transform", using Eq. (28), the path $L_{1}$ into an equivalent sub-path of length $u\left(L_{1}\right) L_{1}$ in the gas in the thermophysical state $\underline{\phi}_{2}$. The two sub-paths in state $\underline{\phi}_{2}$ can then be combined. This 
allows replacing the non-uniform path $L_{1}+L_{2}$ by an equivalent uniform one in the gas at $\underline{\phi}_{2}$. The total length of this equivalent path is $L_{2}+u\left(L_{1}\right) L_{1}$.

$2 /$ then evaluate the transmissivity of the equivalent uniform path at $\underline{\phi_{2}}$ using Eq. (26).

The only difficulty to apply Eq. (26) is to find $u\left(L_{1}\right)$, viz. to solve the implicit equation Eq. (28). We will show, in the next section, how results from the $k$-moment approach can be used to solve this problem.

\subsubsection{Mathematical treatment of Eq. (28)}

Our objective is to find $u\left(L_{1}\right)$ as the solution of Eq. (28).

We can first notice that:

$$
\tau_{1}^{\Delta \eta}\left(L_{1}\right)=\tau_{2}^{\Delta \eta}\left[u\left(L_{1}\right) L_{1}\right] \Leftrightarrow \alpha_{1}^{\Delta \eta}\left(L_{1}\right)=\alpha_{2}^{\Delta \eta}\left[u\left(L_{1}\right) L_{1}\right]
$$

Now if we use Eq. (17) from the previous section, we obtain from Eq. (29):

$$
\alpha_{1}^{\Delta \eta}\left(L_{1}\right)=G r_{2}\left[\alpha_{2,\langle 2\rangle}^{\Delta \eta}\left(u\left(L_{1}\right) L_{1}\right)\right]
$$

in which an explicit dependency of the rank transmutation mapping function with the thermophysical state $\underline{\phi}_{2}$ - subscript " 2 " - is used. In Eq. (30), $\alpha_{2,\langle 2\rangle}^{\Delta \eta}$ represents the second order $k$ moment model - Eq. (14-b) - for the gas in the thermophysical state $\underline{\phi}_{2}$ - we remind that in this section, all the quantities are related to this second order model, as said at the end of the previous Part -.

This allows writing:

$$
\alpha_{2,\langle 2\rangle}^{\Delta \eta}\left[u\left(L_{1}\right) L_{1}\right]=G r_{2}^{-1}\left[\alpha_{1}^{\Delta \eta}\left(L_{1}\right)\right]
$$

where $G r^{-1}$ is the inverse of $G r$.

Finally, using Eq. (13):

$$
u\left(L_{1}\right) L_{1}=\Lambda_{2}\left\{G r_{2}^{-1}\left[\alpha_{1}^{\Delta \eta}\left(L_{1}\right)\right]\right\}
$$

where $\Lambda_{2}$ can be evaluated analytically following its definition, Eq. (12-b).

This allows proposing the following simple method to solve Eq. (28):

1/ evaluate the absorptivity of the uniform path $L_{1}$ - this defines the LHS in Eq. (30) -;

2/ apply the inverse of function $G r_{2}$ - associated with the thermophysical state $\underline{\phi}_{2}$ - to the previous value of absorptivity - see Eq. (31) - . As for the direct function, values of $G r_{2}^{-1}$ can be evaluated first and stored in Tables;

3/ apply Eq. (12-b) - that provides an explicit formula for $\Lambda_{2}$ - to the result obtained at the second step. The result is then $u\left(L_{1}\right) L_{1}$, as shown in Eq. (32). 
4/ as soon as $u\left(L_{1}\right) L_{1}$ is known, the transmissivity of the non-uniform path can be obtained by evaluating the transmissivity of a gas path in state $\underline{\phi}_{2}$ with total length $L_{2}+u\left(L_{1}\right) L_{1}$. This is a direct application of Eq. (26).

3.3. Extension of the technique to more than two sub-paths - the Iterative Copula Estimation (ICE) sche me

\subsubsection{Principle of the ICE scheme}

Eq. (26) can be extended to any number of random variables - viz. to any number of uniform elements - . This provides in this case for $m$ sub-paths, if we define $\underline{\phi}^{\text {ref }}$ as a reference state:

$$
\tau_{1 . . M}^{\Delta \eta}\left(L_{1}, L_{2}, . ., L_{M}\right)=\frac{1}{\Delta \eta} \int_{\Delta \eta} \exp \left\{-\kappa_{\eta}\left(\underline{\phi}^{r e f}\right) \sum_{m=1}^{M} \bar{L}_{r e f}\left[\tau_{m}^{\Delta \eta}\left(L_{m}\right)\right]\right\} d \eta
$$

where $\bar{L}_{r e f}$ is the inverse function of $\tau_{r e f}^{\Delta \eta}(L): \bar{L}_{r e f}\left[\tau_{r e f}^{\Delta \eta}(L)\right]=L$.

Eq. (33) can be seen as some generalization of the Curtis-Godson approximation. More details are given in Appendix C.

The main problem with the previous relationship is that it requires the definition of a reference state. It is known, from $k$-distribution theory, that defining such a reference is not obvious and that it is very difficult to propose unambiguous recommendations to make this choice. Accordingly, in order to circumvent this difficulty we propose to define "local references", that is to say that we suggest modifying the reference state all along the path travelled by the radiative field. This is the principle of the ICE scheme.

In order to explain this method in a simple way, let us consider the case plotted in Figure 2-b. We assume that at the (n-1)-th step of the propagation of the radiation along the path, the transmissivity - or absorptivity - of the gas path between points $\mathrm{M}_{n-1}$ and $\mathrm{M}_{0}$, ie $\alpha_{1 . . n-1}^{\Delta \eta}\left(L_{1}, . ., L_{n-1}\right)$, is known. It is then - by simply iterating the process described previously for two paths - represented as an equivalent uniform path of length $L_{1 . . n-1}$ inside the gas in state $\underline{\phi}_{n-1}$ viz. $\alpha_{1 . n-1}^{\Delta \eta}\left(L_{\mathrm{p}} . ., L_{n-1}\right)=\alpha_{n-}^{\Delta \eta}\left(L_{1 n-}\right)_{1}$. Applying the same idea as for the previous case, with only two subpath, this equivalent uniform path can be transformed into a new equivalent uniform path in thermophysical state $\underline{\phi}_{n}$ with a length $u\left(L_{1 . . n-1}\right) L_{1 . . n-1}$. This then allows calculating the transmissivity from point $\mathrm{M}_{n}$ to $\mathrm{M}_{0}$ as:

$$
\tau_{1 \ldots n}^{\Delta \eta}\left(L_{1}, L_{2}, . ., L_{n}\right)=C_{n}\left[\tau_{n}^{\Delta \eta}\left(L_{n}\right), \tau_{12 \ldots n-1}^{\Delta \eta}\left(L_{1}, L_{2}, . ., L_{n-1}\right)\right]
$$

or more directly:

$$
\tau_{1 \ldots n}^{\Delta \eta}\left(L_{1}, L_{2}, \ldots, L_{n}\right)=\frac{1}{\Delta \eta} \int_{\Delta \eta} \exp \left[-\kappa_{\eta}\left(\underline{\phi}_{n}\right)\left(L_{n}+u\left(L_{1 . . n-1}\right) L_{1 \ldots n-1}\right)\right] d \eta
$$

where, as a generalization of Eq. (32):

$$
u\left(L_{1 . . n-1}\right) L_{1 . . n-1}=\Lambda_{n}\left\{G r_{n}^{-1}\left[\alpha_{1 . . n-1}^{\Delta \eta}\left(L_{1}, L_{2}, . ., L_{n-1}\right)\right]\right\}=\Lambda_{n}\left\{G r_{n}^{-1}\left[\alpha_{n-1}^{\Delta \eta}\left(L_{1 . . n-1}\right)\right]\right\}
$$

This provides a new equivalent uniform path in state $\underline{\phi}_{n}$ of total length $L_{1 . . n}=L_{n}+u\left(L_{1 . . n-1}\right) L_{1 . . n-1}$ and the process can then be iterated. 


\subsubsection{The ICE scheme in practice}

Although the process described in the previous section may look complicated at first sight, it is in fact straightforward to apply. Indeed, calculating the absorptivity of a non-uniform path consists in the following steps:

1/ evaluate the absorptivity of the first column - index 1 -;

2/ apply the inverse mapping $G_{2}^{-1}$ and then function $\Lambda_{2}$ calculated at the thermophysical state of the second column $\underline{\phi}_{2}$. This provides a length $u\left(L_{1}\right) L_{1}$ - see Eq. (36) with $n=2$ or equivalently Eq. (32) -;

3/ calculate the absorptivity of the path $L_{2}+u\left(L_{1}\right) L_{1}$ using the data associated with the thermophysical state of the second column $\underline{\phi_{2}}$.

4/ the column $L_{1}+L_{2}$ can then be treated as a "new first homogeneous column" of length $L_{12}=L_{2}+u\left(L_{1}\right) L_{1}$ in the thermophysical state $\underline{\phi}_{2}$. The process can then be iterated - viz. go back to step 2 - by considering state $\underline{\phi}_{3}$ instead of $\underline{\phi}_{2}$ - ie replace all indices " 2 " by "3" -, then $\underline{\phi}_{4}$ and so on. This procedure continues until the total path is treated.

This process propagates the information about the dependencies between the random lengths in a step by step manner all along the path travelled by the radiation field. It involves, at each new step, the application of an Archimedean copula - see Eqs. (26-28) - that is evaluated at the local thermophysical state. Its accuracy is assessed in the next section, dedicated to applications of the developments described previously.

\section{APPLICATIONS}

\subsection{Model parameters}

The Line-By-Line data used in the present work were extensively described in Ref. [17]. The interested reader should refer to this paper for additional details. They are based on CDSD-4000 [20] for $\mathrm{CO}_{2}$ and HITEMP2010 [21] for $\mathrm{H}_{2} \mathrm{O}$. For comparison purpose, this set of LBL data was also used to generate a $\mathrm{C}-k$ model with 16 values of absorption coefficient. This model uses a Gauss-Legendre quadrature.

From these LBL spectra, we can construct an $l$-distribution model database using the following steps:

1/ for any narrow band (the same narrow bands as described in Ref. [7] were used here), we first start by calculating two mean absorption coefficients directly from the LBL dataset:

- The Planck mean absorption coefficient for each temperature (and $\mathrm{H}_{2} \mathrm{O}$ molar fraction in the case of water vapor):

$$
k_{P}=\frac{1}{\Delta \eta} \int_{\Delta \eta} \kappa_{\eta} d \eta
$$

- The Rosseland mean absorption coefficient for the same set of thermophysical states:

$$
k_{R}=\left(\frac{1}{\Delta \eta} \int_{\Delta \eta} \frac{d \eta}{\kappa_{\eta}}\right)^{-1}
$$

Choosing to represent the $k$-moment model at order 2 in terms of these two coefficients instead of the more natural first and second moments of the absorption coefficient is justified by the so-called 
Inverse Gaussian symmetry property [22] of the second order $k$-moment model. An estimate of $k_{P}$ and $k_{R}$ in any thermophysical state can be obtained by simply interpolating linearly - with respect to temperature (for $\mathrm{CO}_{2}$ ) or temperature plus composition (for $\mathrm{H}_{2} \mathrm{O}$ ) - the values stored in the database. Coefficient $\beta$ that appears in Eq. (14-b) is then given as:

$$
\beta=\pi\left(\frac{k_{P}}{k_{R}}-1\right)^{-1}
$$

The next step is to generate functions $G r$.

Interval $[0,1]$ is divided into $10^{3}$ equally spaced values and for each of them, $G r$ is evaluated directly from its definition - see Eq. (17) - . Function $G r^{-1}-$ which is required in non-uniform situations - is also evaluated using the same discretization scheme. $G r$ and $G r^{-1}$ are estimated for the same set of thermophysical states as $k_{P}$ and $k_{R}$.

These values are stored in Tables. The total size of the Table is 484.8 Mbytes for $\mathrm{CO}_{2}$ (for 48 temperatures between $300 \mathrm{~K}$ and $5000 \mathrm{~K}$ with a $100 \mathrm{~K}$ step) and 4.72 Gbytes for $\mathrm{H}_{2} \mathrm{O}$ (for the same set of temperatures but also for $7 \mathrm{H}_{2} \mathrm{O}$ molar fractions: 0.01, 0.1, 0.2, 0.4, 0.6, 0.8, 1.0).

The calculation of values of $G r$ and $G r^{-1}$ for any thermophysical state and any $X$ value can be performed by using a multi-linear interpolation scheme between the values stored in the Tables: with respect to the gas temperature and $X$ for $\mathrm{CO}_{2}$, with an additional interpolation with respect to the gas molar fraction for $\mathrm{H}_{2} \mathrm{O}$.

\subsection{Model assessment}

\subsubsection{Uniform cases}

The first two cases concern uniform gaseous mixtures of $\mathrm{CO}_{2}$ and $\mathrm{H}_{2} \mathrm{O}$. Mixtures properties are treated, as usual, by considering that the radiative properties of the absorbing species are uncorrelated $[1,2]$.

The first case - C1, Figure 3 - corresponds to Figure 11 from Ref. [7]. It only involves interpolation of the mapping functions with respect to $X$, as all the other quantities $\left(10 \% \mathrm{CO}_{2}, 20 \% \mathrm{H}_{2} \mathrm{O}\right.$, gas temperature is $2000 \mathrm{~K}$ and total pressure is $1 \mathrm{~atm}$.) are inside the database. The second case $-\mathrm{C} 2$, Figure 4 - is almost the same as $\mathrm{C} 1$ but the gas temperature $(2350 \mathrm{~K})$ and molar fraction of $\mathrm{H}_{2} \mathrm{O}$ (0.3) are not inside the database: this case involves a triple interpolation scheme of $\mathrm{Gr}$ functions for $\mathrm{H}_{2} \mathrm{O}$. The molar fraction of $\mathrm{CO}_{2}$ is 0.15 . The length of the gas path is $50 \mathrm{~cm}$ and total pressure is 1 atm.

In both cases, e-distribution model provides a very high accuracy - notice that the absolute differences are represented in logarithmic scale -. These values can be compared, in case $\mathrm{C} 1$, with those given for the SNB model and described in Ref. [7]. In this reference, errors up to 0.02 are reported. In Case $\mathrm{C} 1$, the $\ell$-distribution approach provides an accuracy that is $2-3$ order of magnitudes higher than the $\mathrm{C}-k$ approach. In case $\mathrm{C} 2$, both models provide similar and very high accuracies - absolute difference are lower than $10^{-3}$ when compared with reference LBL calculations -.

In terms of computational cost, it takes 1.47 seconds on one core of an INTEL Xeon CPU E5620 at $2.4 \mathrm{GHz}$ to evaluate $10^{6}$ values of absorptivity per narrow band (bands over which both $\mathrm{CO}_{2}$ and $\mathrm{H}_{2} \mathrm{O}$ absorb) in the case of the C- $k$ model $\ell$-distributions require 0.23 seconds for the same calculation and LBL more than 200 seconds. 


\subsubsection{Non-uniform cases}

Case C3 was adapted from Ref. [19]. It consists of a non-uniform path with the same molar fractions of $\mathrm{H}_{2} \mathrm{O}(0.2)$ and $\mathrm{CO}_{2}(0.1)$ in both columns. The hot layer is at $2000 \mathrm{~K}$, the cold one at $300 \mathrm{~K}$. Both layers have a length of $50 \mathrm{~cm}$. Results - in terms of absorptivity spectrum. Total intensities are also given - are depicted in Figure 5.

The second non-uniform case was taken from Ref. [23]. It corresponds to case $\mathrm{C} 7$ from this reference: gas temperature, species concentrations in $\mathrm{H}_{2} \mathrm{O}$ and $\mathrm{CO}_{2}$ follow Gaussian profiles - the minimum temperature is $300 \mathrm{~K}$ and the maximum temperature, which is attained at the center of the profile, is $1800 \mathrm{~K}$. The minimum and maximum molar fractions of $\mathrm{CO}_{2}$ and $\mathrm{H}_{2} \mathrm{O}$ are 0.1 and 0.9 respectively. The $\mathrm{H}_{2} \mathrm{O}$ molar fraction reaches its minimum at the center and the sum of the two molar fractions is 1.0 all over the path. The continuous profiles were divided into 800 columns each column has a length of $0.1 \mathrm{~cm}$. The total path length is $80 \mathrm{~cm}-$. Results are shown in Figure 6.

It can be noticed that, in both cases C3-4, results are very similar: the correlated- $k$ and PDS/ICE scheme provide comparable errors.

In terms of computational cost, for $10^{4}$ evaluations - in case $\mathrm{C} 4$-, it takes 8.6 seconds per band (bands over which both $\mathrm{CO}_{2}$ and $\mathrm{H}_{2} \mathrm{O}$ absorb) for the $\mathrm{C}-k$ model and 4.6 seconds for $\ell$-distributions on the same processor as for cases $\mathrm{C} 1-2$.

\subsubsection{Turbulence-Radiation Interaction}

The final test case consists of a TRI calculation. The input data contain $10^{5}$ profiles - one thermophysical field every $5.10^{-5}$ second - and all profiles are discretized into 23 uniform columns. These data were provided by Professor P. Coelho from Instituto Superior Tecnico, Lisbon. The method to generate the profiles was described in Ref. [24] and uses the stochastic model for TRI developed in Ref. [25]. The input data required to evaluate the stochastic profiles were taken from http://www.ca.sandia.gov/TNF/DataArch/FlameD.html. The calculation corresponds to Sandia Flame D - methan-air flame -, the radial profile considered passes near the tip of the flame at the location $x=0.45 D$, where $D$ is the diameter of the fuel nozzle.

This test case is appealing for many reasons: $1 /$ this kind of calculation may be challenging because it requires evaluating a large number of distinct spectra $\left(10^{5}\right), 2 /$ the profiles involved are nonuniform, both in terms of temperature and species concentrations and evolve over time.

We have depicted in Figure 7 - top of the Figure - : 1/ the intensity spectrum for the mean field temperature and species concentrations over each uniform path were time-averaged,$- 2 /$ the mean spectrum obtained by calculating one spectrum at each time step and then by averaging the $10^{5}$ instantaneous spectra. There are obvious differences between the spectra that show the effect of TRI on the radiative intensities. Mean relative errors - averaged over the full spectrum, from $50 \mathrm{~cm}^{-1}$ up to $11250 \mathrm{~cm}^{-1}$ - are 0.002 for $\mathrm{C}-k, 0.007$ for $\ell$-distribution, and 0.02 for C- $k 7$ (C- $k$ model with 7 absorption coefficients) when assessed against LBL reference calculations of mean (time-averaged) spectra. Computational time per band (for $10^{5}$ spectra) is 2.85 seconds for C- $k, 1.14$ seconds for $\ell$ distributions and 1.45 seconds for C- $k 7$. For the same case, LBL calculations require more than 410.0 seconds per band (bands over which both $\mathrm{CO}_{2}$ and $\mathrm{H}_{2} \mathrm{O}$ absorb).

\subsection{Discussion}

From the previous sections, the following conclusions can be drawn:

$1 / \ell$-distribution and $k$-distribution models provide a similar accuracy, when assessed against reference LBL calculations, both in uniform and non-uniform media.

$2 /$ in any case, $\ell$-distributions were found to be less expensive in terms of computational cost than $k$ distributions. 
The only defect of $\ell$-distribution modeling, in its present form, is that it is expensive in terms of memory space. Indeed, as noticed earlier, the total size of the database - to treat mixtures of $\mathrm{CO}_{2}$ and $\mathrm{H}_{2} \mathrm{O}$ - is approximately 5 Gbytes - 100 times the size of the C- $k$ database with 16 absorption coefficients -. This explains why all the results - computation times - were given here on a band basis, as our computer was not able to handle the full database due to a lack of RAM. Furthermore, it can be noticed that the approach is formulated in absorptivity / transmissivity form, which mostly restricts its range of applicability to ray tracing / Monte Carlo techniques. However, for applications that require line-of-sight models with simultaneously a high accuracy over narrow spectral bands and a high computational speed, such as encountered for instance in atmospheric studies - analysis of spectral data acquired by satellites - or flame emission spectroscopy, the present approach appears to be a relevant candidate.

$\ell$ - and $k$-distribution methods share many similarities. Indeed, they are both techniques to "summarize" LBL data into compact sets of values. But if $k$-distributions are formulated in terms of absorption coefficients, $\ell$-distribution use absorptivities. In both cases, a probabilistic framework is involved to allow the use of some mathematical tools, useful to develop rigorous mathematical derivations. Both methods then require additional assumptions - scaling, correlation, etc - to extend the approach from uniform to non-uniform situations. In each case, this involves an implicit equation. These equations are funded on dependency models that appear naturally, as soon as the problem is formulated in a probabilistic framework.

\subsubsection{Connection with CDPC models}

All the ideas presented in this paper can be formulated in terms of Cutteridge-Devyatov Polynomial Chaos. More information on this technique can be found in uniform media in Ref. [18] and in nonuniform media in Ref. [26]. Indeed, instead of tabulating the mapping functions, for instance, it is possible to represent $G r$ as polynomials. In this case, databases with smaller size can be constructed. However, from a computational time perspective, using polynomials is more demanding than the simple linear interpolations used here.

Furthermore, as noticed in Ref. [26], the use of the CDPC model in dimensions higher than 2 provides a non-parametric representation of the copula. This formulation does not require any simplify ing as sumption - of path dependent scaled spectra, for instance -. It is thus likely to provide more accurate results than the Archimedean model used here. However, extending the CDPC approach in high dimensions is not as simple as Archimedean copulas due to the so-called curse-ofdimensionality issue [26].

One possibility to increase the accuracy of the model presented here consists in combining the two approaches: Archimedean and non-parametric copula models. Investigating this kind of technique usually referred to as hierarchical or nested copula modeling [27] - is scheduled as future work.

\subsubsection{Copula models in $k$-distribution approaches}

The concept of copula is not restricted to $\ell$-distribution models. They can be readily used together with usual $k$-distribution approaches. The fundamental equation to introduce copulas in $k$ distribution modeling is the following extension of this method to high dimensions [17]:

$$
\left\{\begin{array}{l}
\tau_{12}^{\Delta \eta}\left(L_{1}, L_{2}\right)=\int_{0}^{+\infty} \int_{0}^{+\infty} \frac{\partial^{2} g\left(k_{1}, k_{2}\right)}{\partial k_{1} \partial k_{2}} \exp \left(-k_{1} L_{1}-k_{2} L_{2}\right) d k_{1} d k_{2} \\
g\left(k_{1}, k_{2}\right)=\mathbb{P}\left[\kappa_{\eta}\left(\underline{\phi}_{1}\right) \leq k_{1}, \kappa_{\eta}\left(\underline{\phi}_{2}\right) \leq k_{2}\right]
\end{array}\right.
$$

The joint density function $\partial^{2} g\left(k_{1}, k_{2}\right) / \partial k_{1} \partial k_{2}$ can then be modeled, following Sklar's theorem, as:

$$
\partial^{2} g\left(k_{1}, k_{2}\right) / \partial k_{1} \partial k_{2}=c\left[g_{1}\left(k_{1}\right), g_{2}\left(k_{2}\right)\right] \partial g_{1}\left(k_{1}\right) / \partial k_{1} \partial g_{2}\left(k_{2}\right) / \partial k_{2}
$$


where $c$ is a copula density [16] and $g_{i}\left(k_{i}\right)=\mathbb{P}\left[\kappa_{\eta}\left(\underline{\phi_{i}}\right) \leq k_{i}\right], i=1,2$.

This result can also be used to give an insight into the usual concept of correlation. Indeed, it can be shown that the usual "correlation" refers in fact to the assumption of comonotonicity of gas spectra. Comonotonicity [16] means that there exists a wavenumber scale - that defines a "germ" random variable $g$ - such that any spectrum can be written as a strictly monotonous function of $g$. In this case, the copula associated with this assumption is the so-called upper Fréchet-Hoeffding bound [16]. The corresponding copula density involves a Dirac Delta function [28]:

$$
\partial^{2} g\left(k_{1}, k_{2}\right) / \partial k_{1} \partial k_{2}=\delta\left[g_{1}\left(k_{1}\right)-g_{2}\left(k_{2}\right)\right] \partial g_{1}\left(k_{1}\right) / \partial k_{1} \partial g_{2}\left(k_{2}\right) / \partial k_{2}
$$

If Eq. (42) is put inside Eq. (40), one finds exactly the usual C- $k$ model:

$$
\begin{aligned}
\tau_{12}^{\Delta \eta}\left(L_{1}, L_{2}\right) & =\int_{0}^{1} \int_{0}^{1} c\left(g_{1}, g_{2}\right) \exp \left[-k_{1}\left(g_{1}\right) L_{1}-k_{2}\left(g_{2}\right) L_{2}\right] d g_{1} d g_{2} \\
& =\int_{0}^{1} \int_{0}^{1} \delta\left(g_{1}-g_{2}\right) \exp \left[-k_{1}\left(g_{1}\right) L_{1}-k_{2}\left(g_{2}\right) L_{2}\right] d g_{1} d g_{2} \\
& =\int_{0}^{1} \exp \left[-k_{1}\left(g_{1}\right) L_{1}-k_{2}\left(g_{1}\right) L_{2}\right] d g_{1} \\
& =\int_{0}^{1} \exp \left[-k_{1}(g) L_{1}-k_{2}(g) L_{2}\right] d g
\end{aligned}
$$

Obviously, the present result extends to dimensions higher than 2.

Introducing copula models in usual $k$-distribution approaches is also likely to provide new possibilities to handle spectral correlations over non-uniform paths, and to improve this approach in cases for which the usual correlation / comonotonicity assumption is known to fail. 


\section{CONCLUSION}

A new approach was presented for radiative heat transfer in uniform and non-uniform gaseous media: the $l$-distribution modeling. The mathematical foundations of the model were described. It is mostly based on the fact that band averaged absorptivities are distribution functions of absorption path lengths. As soon as a probabilistic framework is used, several tools from this area can be used: rank transmutation mapping, to extend the $k$-moment method to an infinite number of moments of the absorption coefficient; copulas to handle path non-uniformities. Formulating the problem in terms of probabilities mostly provides a convenient way to "summarize" LBL data into compact sets and to extend the approach from uniform to non-uniform situations: this idea is shared by both $k$ - and $l$-distribution models.

The new approach was shown to be in the worst cases as accurate as the usual C-k model, both in uniform and non-uniform media. Its other advantage is its computational cost that was found to be lower than $k$-distribution models. Its main defect is that it requires, in its present form, a large amount of memory space. Additional work is required in order to reduce the size of the databases.

All the codes and databases (C- $k, \ell$-distributions) are available on request for evaluation purpose contact: frederic.andre@ insa-lyon.fr -.

\section{AKNOWLEDGEMENTS}

The author would like to thank Professor Pedro COELHO, from Instituto Superior Tec nico, Lisbon, for providing the TRI data used in Section 4.2.3.

\section{REFERENCES}

[1] GOODY R. M., YUNG Y. L. Atmospheric Radiation: Theoretical Basis, Oxford University Press, 1989.

[2] TAINE J, SOUFIANI A. Gas IR radiative properties: From spectroscopic data to approximate models, Adv. Heat Transfer 1999;33:295-414.

[3] ANDRE F., VAILLON R., A simple and accurate method to derive SNB parameters for the radiative properties of gases. In: Lemonnier D, Selçuk N, Lyabaert P, Editors. Proceedings of Eurotherm78-Computational thermal radiation in participating media II, Poitiers. Lavoisier, Paris; 5-7 avril, 2006: 167-176.

[4] ANDRE F, VAILLON R. The $k$-moment method for modeling the blackbody weighted transmission function for narrow and wide band radiative properties of gases, JQSRT 2007;108:116.

[5] ANDRE F., VAILLON R., Generalization of the $k$-moment method using the maximum entropy principle. Application to the NBKM and full spectrum SLMB gas radiation models, JQSRT 2007;113:1508-1520.

[6] MALKMUS W. Random Lorentz band model with exponential-tailed S-1 line-intensity distribution function, J. Opt. Soc. Am 1967; 57:323-329.

[7] RIVIERE Ph., SOUFIANI A. Updated band model parameters for $\mathrm{H}_{2} \mathrm{O}, \mathrm{CO}_{2}, \mathrm{CH}_{4}$ and $\mathrm{CO}$ radiation at high temperature, IJHMT 2012;55:3349-3358.

[8] SCHORACK G.R. Probability for statisticians, Springer texts in statistics, Springer, 2000.

[9] DUNN, W.L., SHULTIS, J.K. Exploring Monte Carlo Methods, Elsevier, 2012. 
[10] SHAW W.T., BUCKLEY I.R.C. The alchemy of probabilistic distributions: behond GramCharlier expansions, and a skew-kurtotic-normal distribution from a rank transmutation map, presented at the First IMA Computational Finance Conference, $23^{\text {rd }}$ March 2007.

[11] ABRAMOWITZ M., STEGUN I.A. Handbook of Mathematical Functions with formulas, Graphs, and Mathematical Tables, New York: Dover 1965.

[12] ANDREF., VAILLON R. The $k$-moment method for the narrow band modeling of radiative properties of nonuniform gaseous media, JQSRT 2008;109:258-268.

[13] ANDRE F, VAILLON R. A non uniform narrow band correlated- $k$ approximation using the $k$ moment method, JQSRT 2010;111:1900-1911.

[14] ANDREF, VAILLON R. The spectral-line moment-based (SLMB) modeling of the wide band and global blackbody-weighted transmission function and cumulative distribution function of the absorption coefficient in uniform gaseous media, JQSRT 2008;109:2401-2416.

[15] SKLAR A. Fonctions de répartitions et leurs marges, Publications of the Institute of Statistics, Université de Paris, 1959;8:229-231.

[16] NELSEN R.B. An introduction to Copulas - Second Edition, Springer series in statistics, Springer, 2006.

[17] ANDRE F, HOU L, ROGER M, VAILLON R. The multispectral gas radiation modeling : a new theoretical framework based on a multidimensional approach to $k$-distribution methods, JQSRT 2014;147:178-195.

[18] ANDRE F., GALTIER M. On the use of polynomial chaos expansions and generalized moments within the frame of gas radiation in non-uniform gaseous media, JQSRT 2015;164:184192.

[19] MODEST MF. Narrow-band and full-spectrum $k$-distributions for radiative heat transfer correlated- $k$ vs. scaling approximation, JQSRT 2003;76:69-83.

[20] TASHKUN SA, PEREVALOV VI. CDSD-4000: High-resolution, high-temperature carbon dioxide spectroscopic databank, JQSRT 2011;112:1403-1410.

[21] ROTHMAN L.S., GORDON L.E., BARBER R.J., DOTHE H., GAMACHE R.R., GOLDMAN A., PEREVALOV V.I., TASHKUN S.A., TENNYSON J., HITEMP, the hightemperature molecular spectroscopic database, JQSRT 2010;111:2139-2150.

[22] MUDHOLKAR G.S., NATARAJAN R. The inverse Gaussian models: analogues of symmmetry, skweness and kurtosis, Ann. Inst. Statist. Math. 2002;54:138-154.

[23] BHARADWAJ S.P., MODEST M.F. A multiscale Malkmus model for treatment of inhomogeneous paths, IJTS 2007;46:479-490.

[24] COELHO P.J. Detailed numerical simulation of radiative transfer in a nonluminous turbulent jet diffusion flame, Combustion and Flame 2004;136:481-492.

[25] CHAN S.H., PAN X.C. A general semi-causal stochastic model for turbulence/radiation interactions in flames, JHT 1997;119:509-516.

[26] ANDRE F. A polynomial chaos approach to narrow band modeling of radiative heat transfer in non-uniform gaseous media, JQSRT, under review.

[27] HOFERT, M. Sampling Archimedean copulas, Computational Statistics and Data Analysis 2008;52:5163-5174.

[28] KREPS, R.E. A partially comonotonic algorithm for loss generation, ASTIN Colloquium Papers 2000, pp 165-176. 
[29] Mc NEIL A., NESLEHOVA J. Multivariate Archimedean copulas, d-monotone functions and 11-norm symmetric distributions, The Annals of Statistics 2009;37:3059-3097.

[30] WIDDER DV. Necessary and sufficient conditions for the representation of a function as a Laplace integral, Transactions of the American Mathematical Society 1931;33:851-892.

[31] KIMBERLING C.H. A probabilistic interpretation of complete monotonicity, Aequationes Math 1974;10:152-164.

[32] OAKES D. Bivariate survival models induced by frailties, Journal of the American Statistical Association 1989;84:487-493.

[33] YOUNG S.J. Non isothermal band model theory, JQSRT 1977;18:1-28. 


\section{FIGURE CAPTIONS}

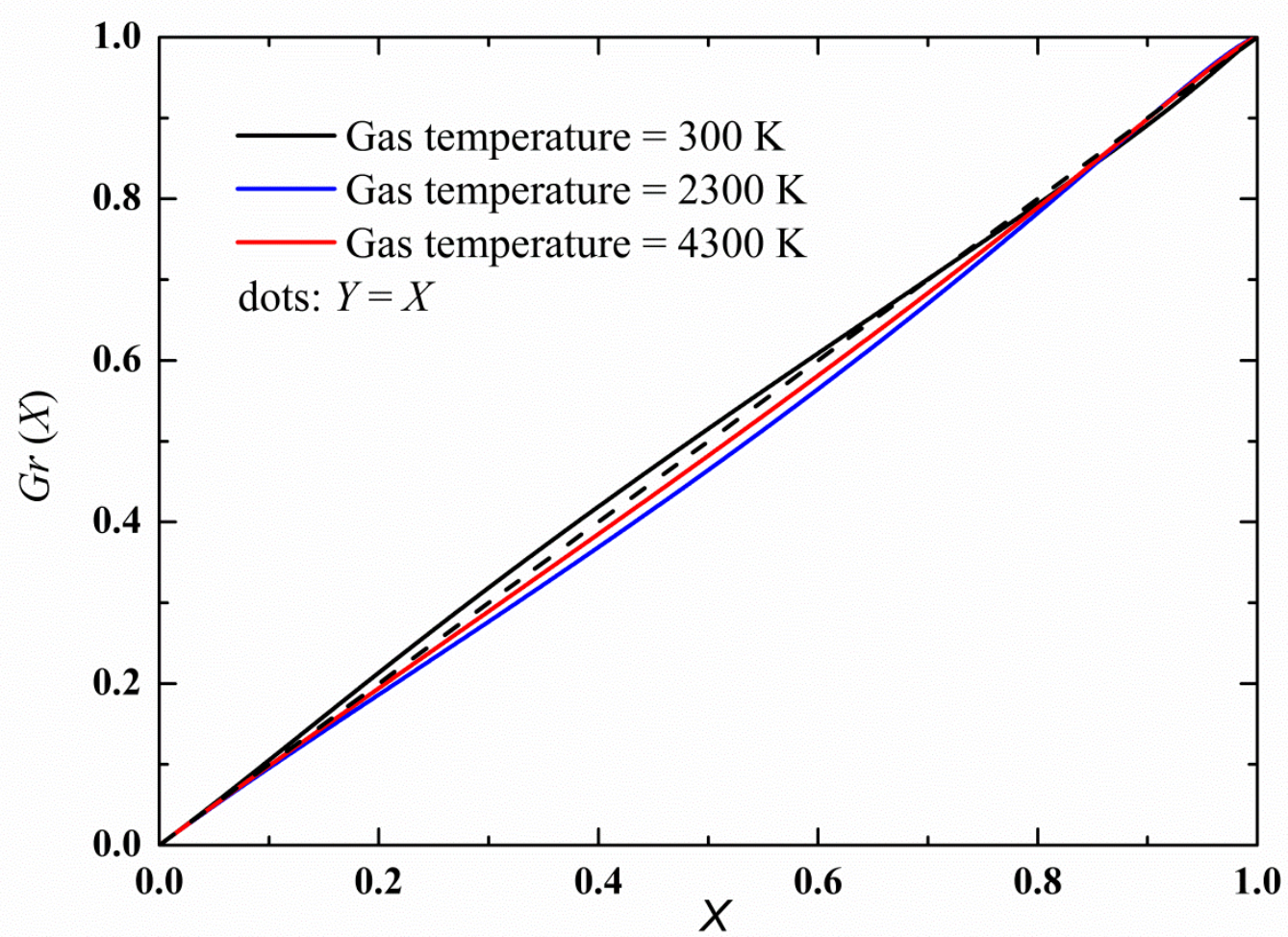

Figure 1. Rank transmutation mapping functions $G r$ for various gas temperatures for $\mathrm{H}_{2} \mathrm{O}$, molar fraction 0.01 , over the $25 \mathrm{~cm}^{-1}$ narrow band centered at $3300 \mathrm{~cm}^{-1}$. 
Non-uniform path

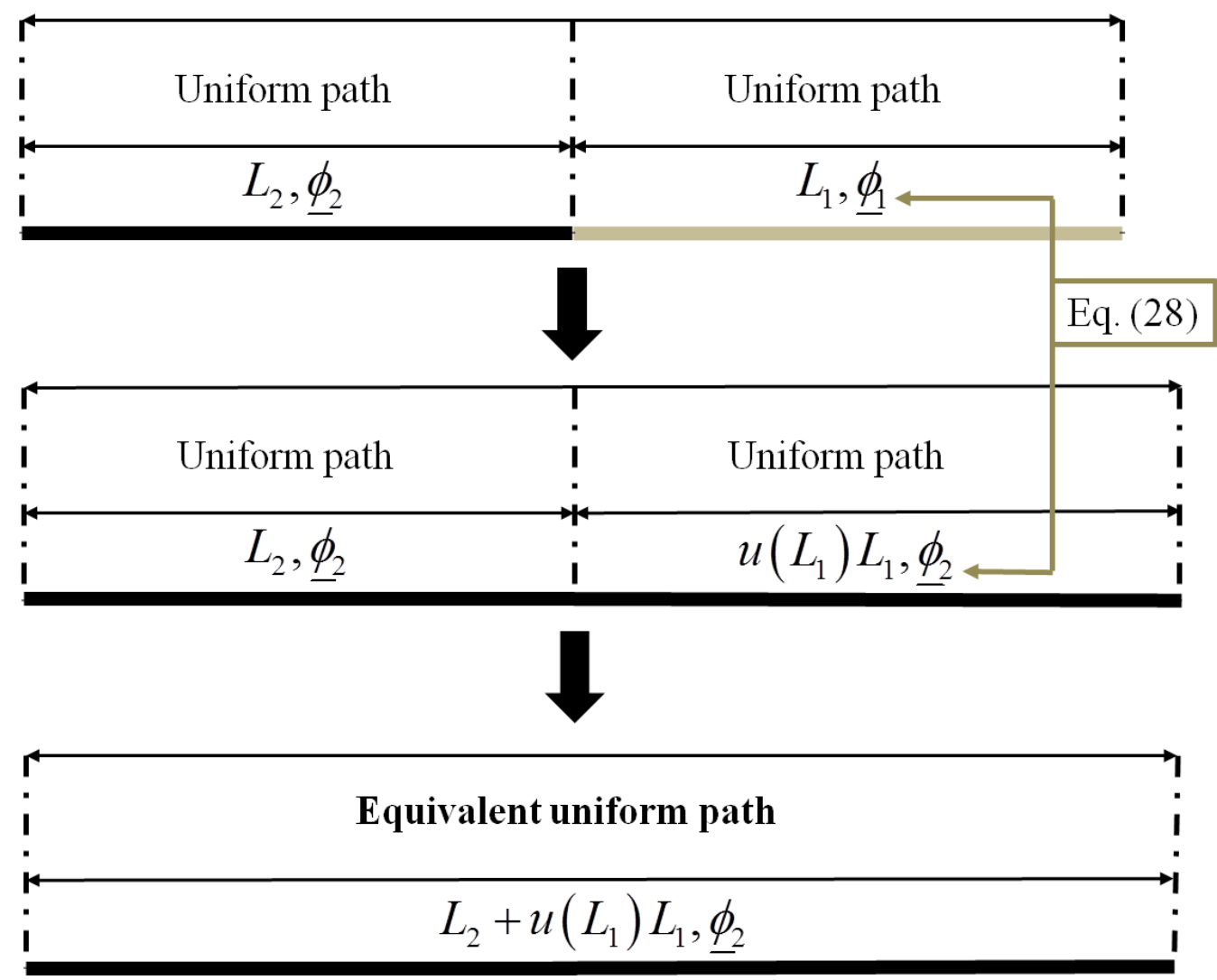

Figure 2-a. Principle of the ICE scheme for two columns. On this figure, the radiation propagates from left to right. 


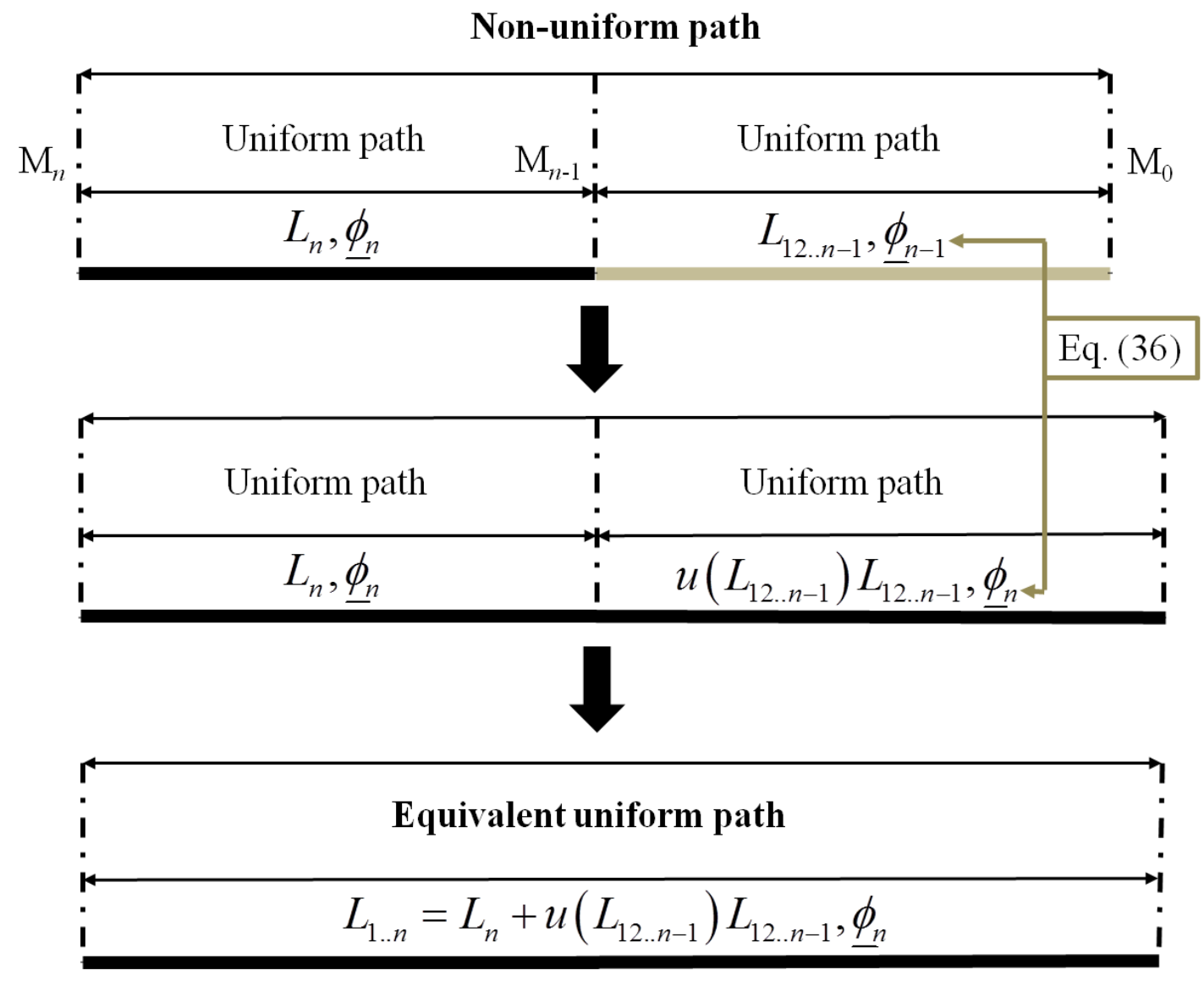

Figure 2-b. Principle of the ICE scheme for more than 2 columns. On this figure, the radiation propagates from left to right. 


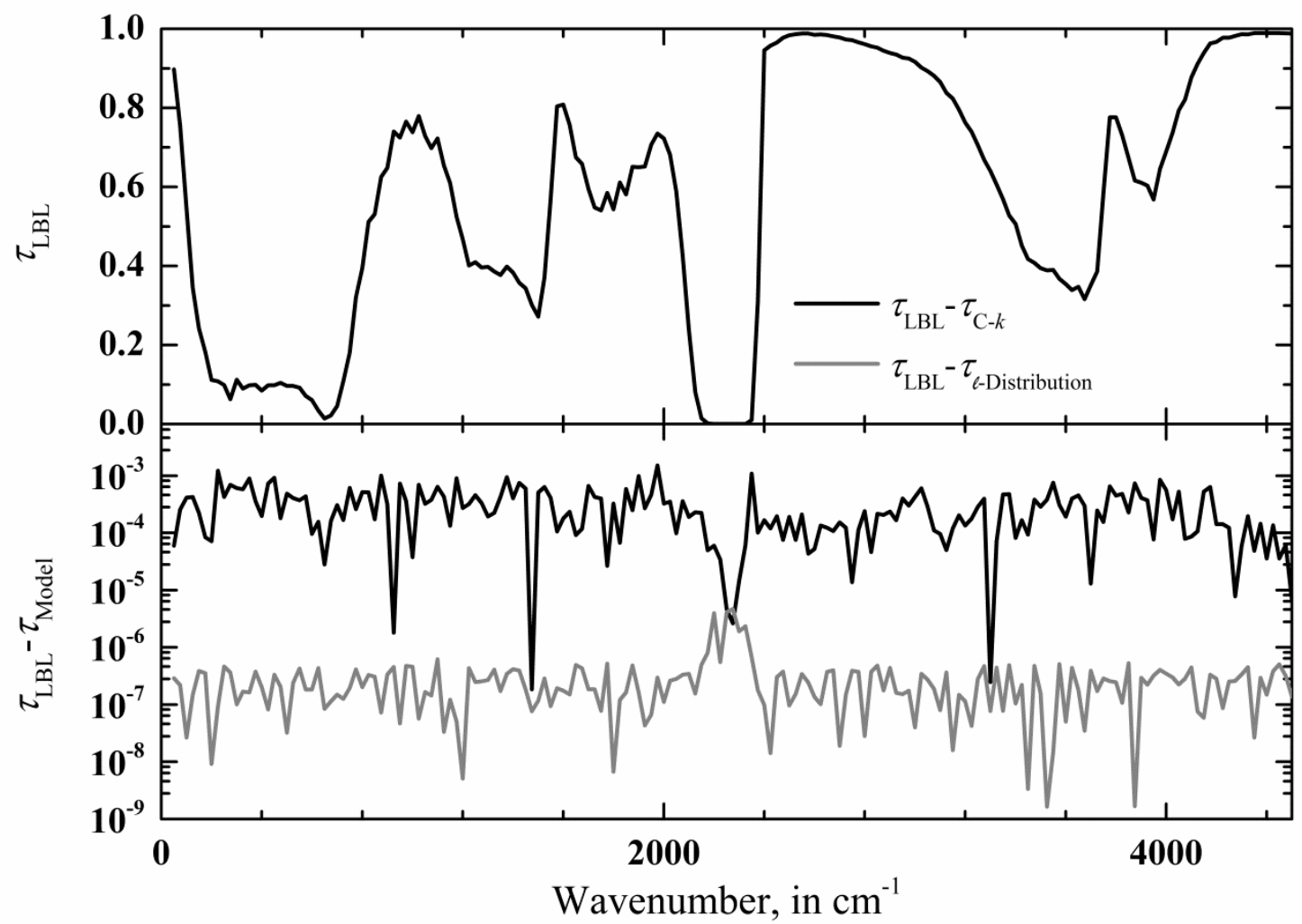

Figure 3. Case $\mathrm{C} 1$ - Narrow band transmissivity for a uniform $10 \% \mathrm{CO}_{2}-20 \% \mathrm{H}_{2} \mathrm{O}-70 \% \mathrm{~N}_{2}$ mixture calculated by the LBL, C- $k$ and $\ell$-distribution models. Gas temperature is $2000 \mathrm{~K}$ and total pressure 1 atmosphere. Total path length is $100 \mathrm{~cm}$. 


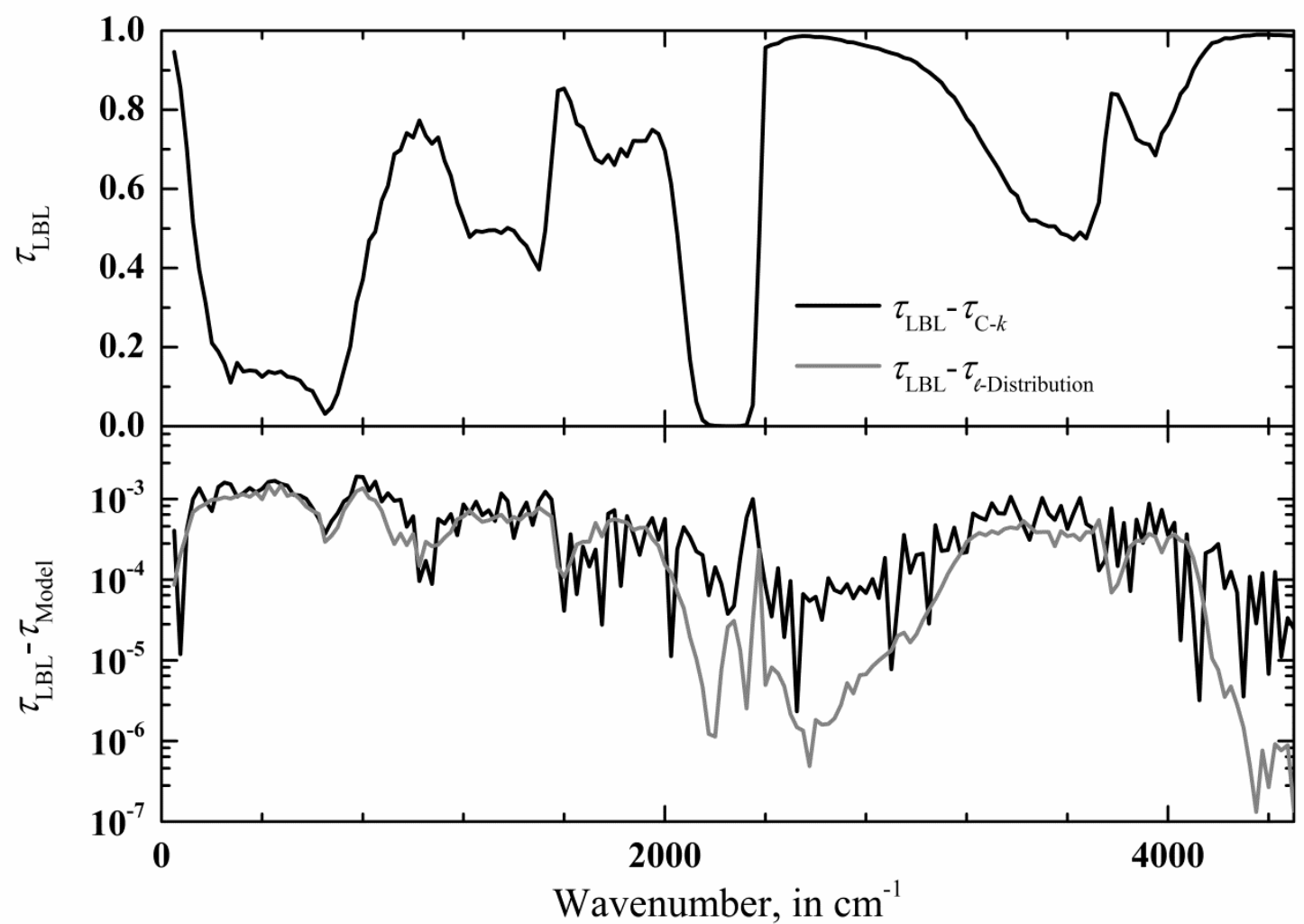

Figure 4. Case $\mathrm{C} 2$ - Narrow band transmissivity for a uniform $15 \% \mathrm{CO}_{2}-30 \% \mathrm{H}_{2} \mathrm{O}-55 \% \mathrm{~N}_{2}$ mixture calculated by the LBL, C- $k$ and $\ell$-distribution models. Gas temperature is $2350 \mathrm{~K}$ and total pressure 1 atmosphere. Total path length is $50 \mathrm{~cm}$. 


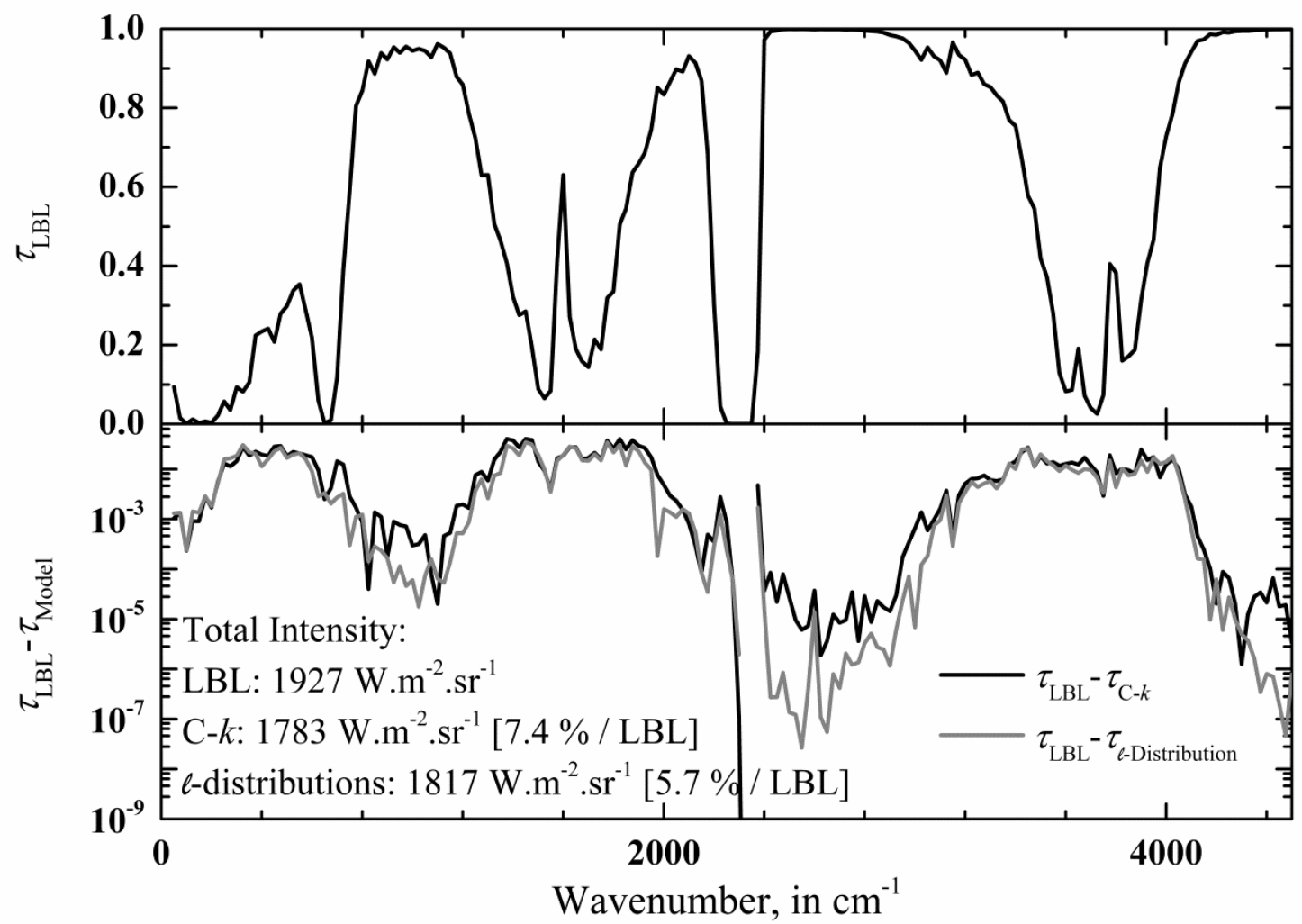

Figure 5. Case $\mathrm{C} 3$ - Narrow band transmissivity for a non- isothermal $10 \% \mathrm{CO}_{2}-20 \% \mathrm{H}_{2} \mathrm{O}-70$ $\% \mathrm{~N}_{2}$ mixture calculated by the LBL, C- $k$ and $\ell$-distribution models. Gas temperature is $2000 \mathrm{~K}$ in the hot column and $300 \mathrm{~K}$ in the cold one. Total pressure is 1 atmosphere. Each isothermal path has a length of $50 \mathrm{~cm}$. 


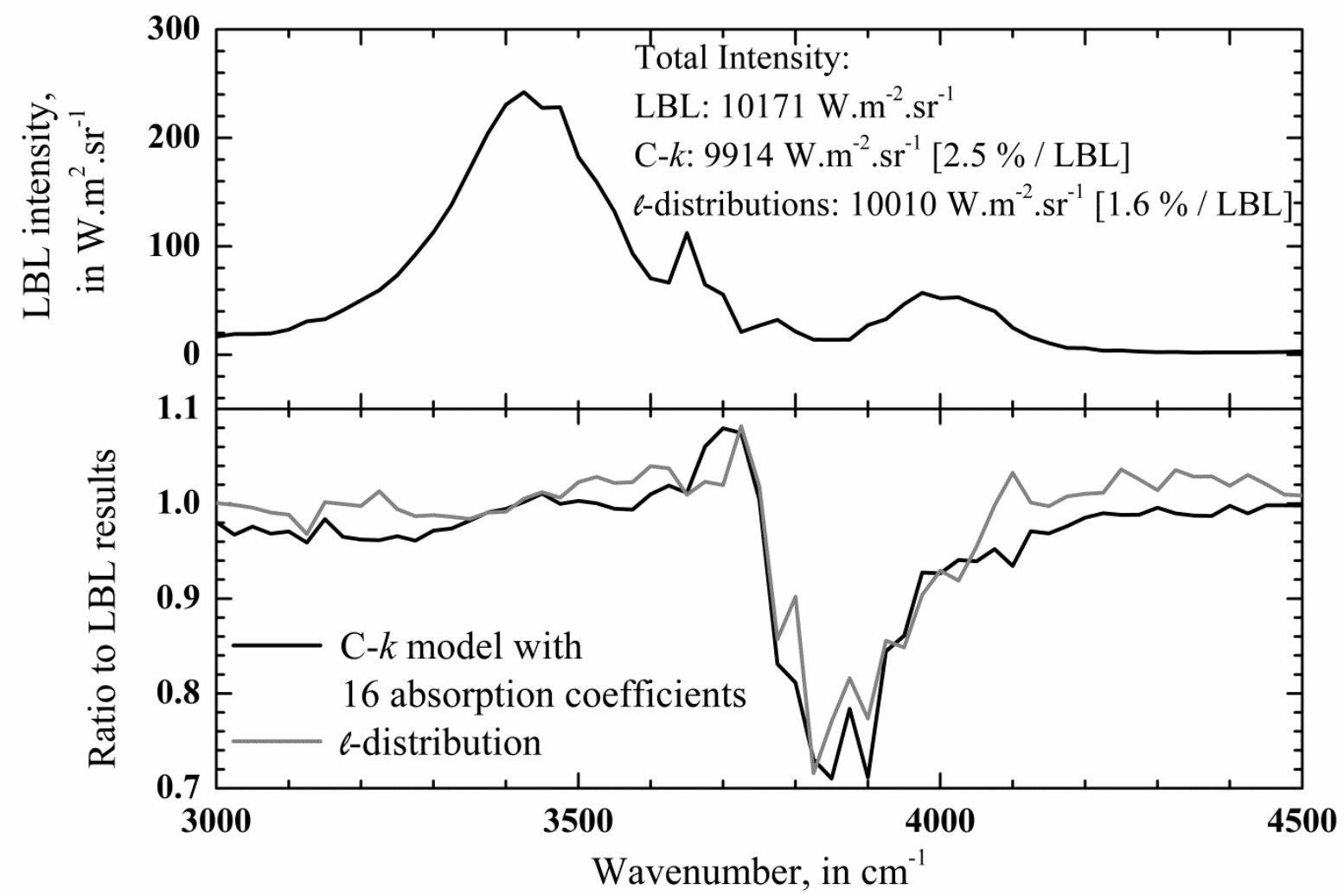

Figure 6. Case $\mathrm{C} 4$ - Narrow band intensity for a non-uniform $\mathrm{CO}_{2}-\mathrm{H}_{2} \mathrm{O}$ mixture calculated by the LBL, C- $k$ and $\ell$-distribution models. Total pressure is 1 atmosphere. Temperature and species concentrations profiles are Gaussian - see text for details -. Total path length is $80 \mathrm{~cm}$. 


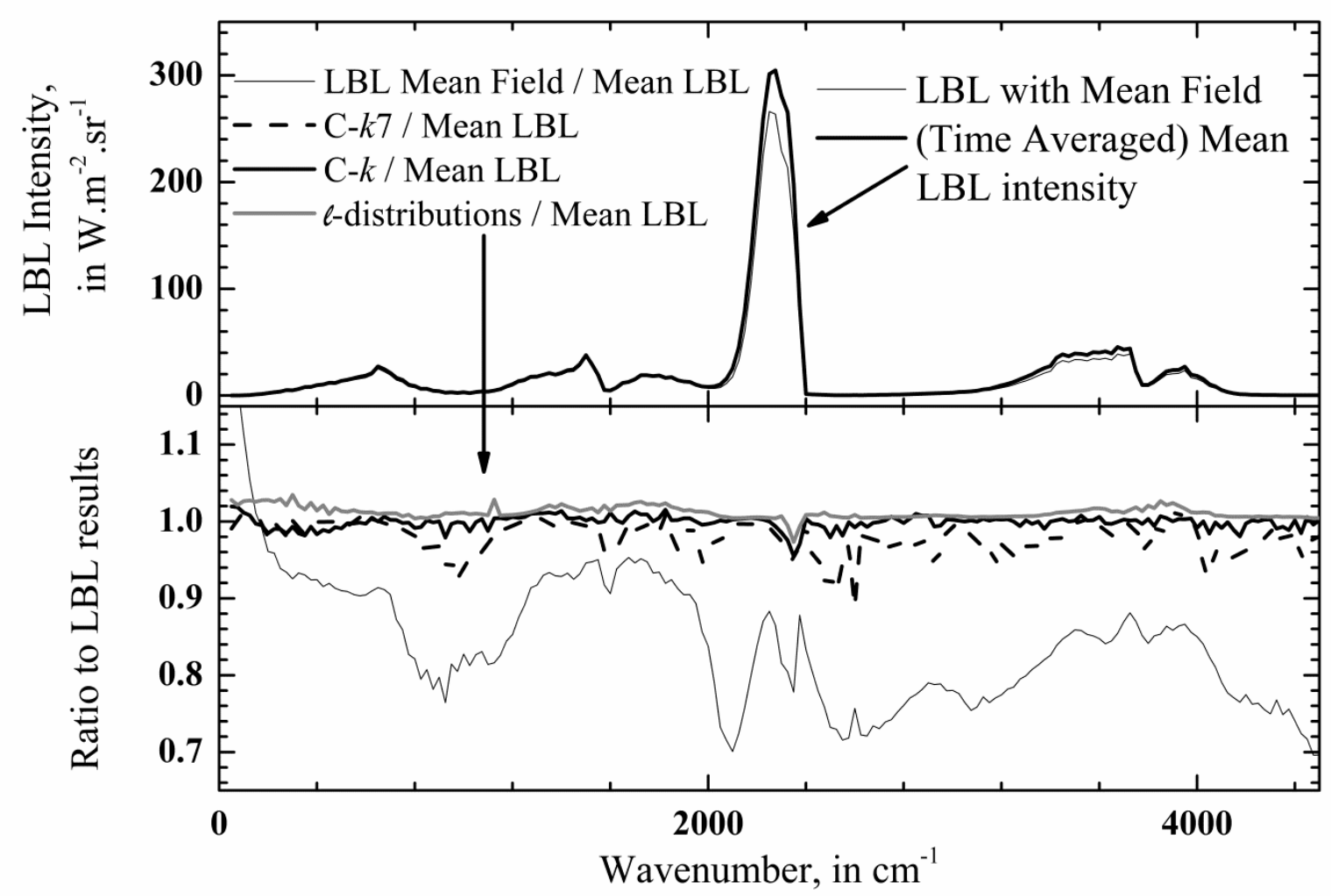

Figure 7. Case C5. Turbulence Radiation Interaction. Top: LBL intensity with the mean - time averaged - temperature and species concentration profiles, Time averaged LBL intensity; bottom: ratio to mean $\mathrm{LBL}$ intensity for the $\mathrm{LBL}$ model with mean field, $\mathrm{C}-k, \mathrm{C}-k 7$ and $\ell$-distribution models. 


\section{APPENDIX A. Short introduction to Archime dean copulas.}

It is out of the scope of the present work to provide a comprehensive depiction of copulas' theory. The interested reader should refer to Ref. [16] for more details on this topic. However, we provide here some general definitions together with some theorems - mostly related to Archimedean copulas - that may be useful to understand the non-uniform approach developed in this work.

A copula in dimension 2: 1/ is a bi-variate cumulative distribution function with uniform marginals; $2 /$ contains all the information about the dependence structure between sets of random variables. It has the following mathematical properties:

- $\quad P 1$ : For every $u \in[0,1]$ and $v \in[0,1], C(u, 1)=u$ and $C(1, v)=v$;

- $\quad P 2$ : For every $u \in[0,1]$ and $v \in[0,1], C(u, 0)=C(0, v)=0$;

- $\quad P 3$ : It is 2-increasing - which in our case simply means that $\frac{\partial^{2} C(u, v)}{\partial u \partial v} \geq 0$ -

More general definitions and further details about copulas can be found in Nelsen [16].

Archimedean copulas are among the most popular ones. Many "usual" copula models are from this family: Clayton, Ali-Mikhail-Haq, etc - see for instance Table 4.1 from Ref. [16] -.

Some useful results about Archimedean copulas are given below.

An Archimedean generator is defined [29] as a strictly decreasing and continuous function $\psi:[0,+\infty] \rightarrow[0,1]$ that satisfies $\psi(0)=1$ and $\psi(+\infty)=0$. Following this definition, under $H 2$, the band averaged transmissivity of the gas in any thermophysical state $\phi_{m}$ is an Archimedean generator.

Furthermore, one can notice that transmissivities are completely monotone functions on $[0,+\infty]$ since for any integer $n \in \mathbb{N},(-1)^{n} \frac{\partial^{n} \tau_{m}^{\Delta \eta}(L)}{\partial L^{n}}=\frac{1}{\Delta \eta} \int_{\Delta \eta}\left[\kappa_{\eta}\left(\underline{\phi}_{m}\right)\right]^{n} \exp \left[-\kappa_{\eta}\left(\underline{\phi}_{m}\right) L\right] d \eta \geq 0$. It can be noticed that complete monotonicity can be used to show that transmissivities are Laplace transforms of some distribution functions - this is known as Bernstein-Widder theorem [30] -. This result can be seen as the mathematical foundation of $k$-distribution's theory. This fact was already emphasized in Ref. [5].

Finally, Kimberling showed in Ref. [31] that for any completely monotone, strictly decreasing, continuous function $\psi:[0,+\infty] \rightarrow[0,1]$ that satisfies $\psi(0)=1$ and $\psi(+\infty)=0$ then, with $(u, v) \in[0,1]^{2}, C(u, v)=\psi\left[\psi^{-1}(u)+\psi^{-1}(v)\right]$ is a copula. This results can also be extended to any dimension $d \geq 2$.

If we replace $\psi$ by $\tau_{2}^{\Delta \eta}$ and compare the result with Eqs. (26-28), we see that the path dependent scaled approximation corresponds rigorously to a dependency model that can be formulated in terms of Archimedean copulas. In this case, $\tau_{2}^{\Delta \eta}$ plays the role of an Archimedean generator. The extension of this result to higher dimensions using a reference state - see Eq. (33) - arises directly from Kimberling's theorem. 
Finally, it can be noticed that, in survival analysis, Archimedean copulas are strongly connected with so-called Frailty models - see Ref. [32] for details -.

Survival Theory (ST) and radiative transfer - and more directly $l$-distribution modeling - share many similarities. Indeed, the main objective of ST is to study random variables that are time-toevents - for instance, the time taken by an individual before to relieve after taking a given drug. Radiative transfer can be seen as a ST applied to quanta. But in this case, the random variable which is the most relevant is not a time but a distance to event as, for instance, a distance for a quantum emitted by a black wall to be absorbed by the medium. This is thus not really surprising to see concepts from ST appear in Gas Radiation modeling, as the two problems are fundamentally the same. 


\section{APPENDIX B. Further details on the Path Dependent Scaling Approximation}

The main defect of the scaled- $k$ approximation is that it does not account for spectral lines that may appear in the spectrum in a given state $\underline{\phi}$ but that are not observed in other states. One possible way to handle this problem consists in writing the spectrum in state $\underline{\phi}$ as:

$$
\kappa_{\eta}(\underline{\phi})=\kappa_{\eta}\left(\underline{\phi}^{r e f}\right)\left(\bar{u}+\delta u_{\eta}\right)
$$

where $\underline{\phi}^{\text {ref }}$ represents a reference state, $\bar{u}$ some mean ratio between the spectra in the two states and $\delta u_{\eta}$ a wavenumber dependent effect that measures the error made by approximating the spectra as truly scaled.

$\bar{u}$ can be obtained - for instance, as this choice is not unique - at the optically thin limit. This provides the two equations:

$$
\left\{\begin{array}{l}
\bar{u}=\frac{k_{P}(\underline{\phi})}{k_{P}\left(\underline{\phi}^{r e f}\right)} \\
\frac{1}{\Delta \eta} \int_{\Delta \eta} \kappa_{\eta}\left(\underline{\phi}^{r e f}\right) \cdot \delta u_{\eta} d \eta=0
\end{array}\right.
$$

Let us now consider a spectral interval $\Delta \eta(k) \subset \Delta \eta$ such that for any $\eta \in \Delta \eta(k)$, $k \leq \kappa_{\eta}\left(\underline{\phi}^{r e f}\right) \leq k+d k$ where $d k$ represents a small interval of $k$-values. Then one can write:

$$
\begin{aligned}
\tau^{\Delta \eta(k)}(l) & =\frac{1}{\Delta \eta(k)} \int_{\Delta \eta(k)} \exp \left[-\kappa_{\eta}(\underline{\phi}) l\right] d \eta \\
& =\frac{1}{\Delta \eta(k)} \int_{\Delta \eta(k)} \exp \left[-\kappa_{\eta}\left(\underline{\phi}^{r e f}\right)\left(\bar{u}+\delta u_{\eta}\right) l\right] d \eta \\
& =\frac{1}{\Delta \eta(k)} \int_{\Delta \eta(k)} \exp \left[-k\left(\bar{u}+\delta u_{\eta}\right) l\right] d \eta \\
& =\exp (-k \bar{u} l) \frac{1}{\Delta \eta(k)} \int_{\Delta \eta(k)} \exp \left[-k \delta u_{\eta}(\underline{\phi}) l\right] d \eta
\end{aligned}
$$

or equivalently:

$$
\tau^{\Delta \eta(k)}(l)=\exp [-k R(k, l)]
$$

in which we have introduced the function:

$$
R(k, l)=\bar{u} l-\frac{1}{k} \ln \left\{\frac{1}{\Delta \eta(k)} \int_{\Delta \eta(k)} \exp \left[-k \delta u_{\eta}(\underline{\phi}) l\right] d \eta\right\}
$$

Now, if we assume that the effect of the non-uniformities can be represented as a path dependent scaling function that does not depend on $\kappa_{\eta}\left(\underline{\phi}^{\text {ref }}\right)$ viz.: 


$$
\frac{\partial R(k, l)}{\partial k}=0
$$

then, any value of the absorption coefficient can be used to define function $R$. This provides, using for instance the Planck mean absorption coefficient $k_{P}\left(\underline{\phi}^{\text {ref }}\right)$ for this purpose:

$$
\left\{\begin{array}{l}
\tau^{\Delta \eta(k)}(l)=\exp \left[-k R\left(k=k_{p}\left(\underline{\phi}^{r e f}\right), l\right)\right]=\exp [-k u(l) l] \\
u(l)=\frac{R\left(k=k_{p}\left(\underline{\phi}^{\text {ref }}\right), l\right)}{l}=\bar{u}-\frac{1}{k_{p}\left(\underline{\phi}^{\text {ref }}\right) l} \ln \left\{\frac{1}{\Delta \eta\left[k_{p}\left(\underline{\phi}^{\text {ref }}\right)\right]} \int_{\Delta \eta\left[k_{p}\left(\underline{\phi}^{\text {ref }}\right)\right]} \exp \left[-k_{p}\left(\underline{\phi}^{\text {ref }}\right) \delta u_{\eta}(\underline{\phi}) l\right] d \eta\right\}
\end{array}\right.
$$

One can notice that when there is no error - ie. $\delta u_{\eta}=0-$ then this approach gives exactly the scaled model. 


\section{Appendix C. The Curtis-Godson approximation revisited}

The aim of the present Appendix is to show that Eq. (33) is closely related to the Curtis-Godson approximation, frequently encountered within the frame of Statistical Narrow Band models. More details about the "usual" way to introduce this approximation can be found in Ref. [33].

First of all, we will start by assuming that: $1 /$ there exists a reference state such that any spectrum can be assumed to be path dependent scaled - see the definition in Section 3.2. - with it, 2/ the rank transmutation mapping functions and their inverse - for each thermophysical state - are defined as $\operatorname{Gr}(X)=X, G r^{-1}(X)=X, X \in[0,1]$. This second assumption simply means that the spectrum follows exactly the assumptions of the SNB model with the Malkmus' distribution of linestrengths. In this case, the second order $k$-moment model - Eq. (14-b) - is exact.

Following Eq. (33), rewritten in terms of $u$ functions - see for instance Eq. (25) - :

$$
\tau_{1 . . M}^{\Delta \eta}\left(L_{1}, L_{2}, . ., L_{M}\right)=\frac{1}{\Delta \eta} \int_{\Delta \eta} \exp \left[-\kappa_{\eta}\left(\underline{\phi}^{r e f}\right) \sum_{m=1}^{M} u\left(L_{m}\right) L_{m}\right] d \eta
$$

Now, as $G_{R}(X)=X, G_{R}^{-1}(X)=X, X \in[0,1]$, we can write over each uniform path $m$-from Eq. (36) - :

$$
u\left(L_{m}\right) L_{m}=\Lambda_{r e f}\left[1-\tau_{m,\langle 2\rangle}^{\Delta \eta}\left(L_{m}\right)\right]=-\frac{\ln \left[\tau_{m,\langle 2\rangle}^{\Delta \eta}\left(L_{m}\right)\right]}{k_{P}\left(\underline{\phi}^{r e f}\right)}+\frac{\omega_{2}\left(\underline{\phi}^{r e f}\right) k_{P}\left(\underline{\phi}^{r e f}\right)}{\omega_{1}^{2}\left(\underline{\phi}^{r e f}\right)}\left\{\frac{\left.\ln \left[\tau_{m,\langle 2\rangle}^{\Delta \eta}\left(L_{m}\right)\right]\right]^{2}}{k_{P}\left(\underline{\phi}^{r e f}\right)}\right\}^{2}
$$

Eq. (C.2) can then be considered at the optically thin and thick limits to yield - these relationships are obtained by simply using the definition of the transmissivity as given by Eq. (14-b) inside (C.2) - :

- at the optically thin limit:

$$
u\left(L_{m}\right) \approx \frac{k_{P}\left(\underline{\phi}_{m}\right)}{k_{P}\left(\underline{\phi}^{r e f}\right)}
$$

- $\quad$ at the optically thick limit:

$$
u\left(L_{m}\right) \approx \frac{\beta\left(\underline{\phi}_{m}\right) k_{P}\left(\underline{\phi}_{m}\right)}{\beta\left(\underline{\phi}^{r e f}\right) k_{P}\left(\underline{\phi}^{r e f}\right)}
$$

that provide, when put inside (C.1), exactly the same equations as used in the Curtis-Godson approximation. Indeed, Eqs. (C.3,C.4) yield:

- at the optically thin limit:

$$
\tau_{1 . . M}^{\Delta \eta}\left(L_{1}, L_{2}, . ., L_{M}\right) \approx \exp \left[-k_{P}\left(\underline{\phi}^{r e f}\right) \sum_{m=1}^{M} u\left(L_{m}\right) L_{m}\right]=\exp \left[-\sum_{m=1}^{M} k_{P}\left(\underline{\phi}_{m}\right) L_{m}\right]
$$

- at the optically thick limit:

$$
\begin{aligned}
\tau_{1 . . M}^{\Delta \eta}\left(L_{1}, L_{2}, . ., L_{M}\right) & \approx \exp \left[-\sqrt{\frac{2}{\pi} k_{P}\left(\underline{\phi}^{\text {ref }}\right) \beta\left(\underline{\phi}^{\text {ref }}\right) \sum_{m=1}^{M} u\left(L_{m}\right) L_{m}}\right] \\
& =\exp \left[-\sqrt{\frac{2}{\pi} \sum_{m=1}^{M} k_{P}\left(\underline{\phi}_{m}\right) \beta\left(\underline{\phi}_{m}\right) L_{m}}\right]
\end{aligned}
$$

This allows defining two equivalent - index $e$ - SNB coefficients - this is the main idea behind the Curtis-Godson approximation [1-2,33] -: 


$$
\begin{gathered}
k_{P, e} \sum_{m=1}^{M} L_{m}=\sum_{m=1}^{M} k_{P}\left(\underline{\phi}_{m}\right) L_{m} \\
k_{P, e} \beta_{e} \sum_{m=1}^{M} L_{m}=\sum_{m=1}^{M} k_{P}\left(\underline{\phi}_{m}\right) \beta\left(\underline{\phi}_{m}\right) L_{m}
\end{gathered}
$$

They are the same as those given in Ref. [33].

Notice, from the previous equation, that as soon as equivalent coefficients are defined, all influence of the model on any reference state disappears. This simply means that when $G r(X)=X, G r^{-1}(X)=X, X \in[0,1]$, the reference state does not need to be defined explicitly.

Accordingly, one of the other advantages of $\ell$-distribution modeling is, from a pedagogical perspective, to propose a different view on the treatment of non-uniformities which is fully compatible with more usual existing techniques. 